Report Issued:

January 8, 1993

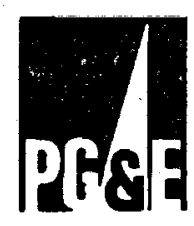

Research and

Development :

\section{Summary of New Generation Technologies and Resources}

\author{
Advanced Energy Systems \\ Report 007.6-92.3 \\ Program Manager: George A. Hay III \\ Technology Assessment, Monitoring, and Scanning (TAMS) \\ FInal Report, December 1992
}

Project Managers: David W. Behrens Donna S. Lindquist Ronnie A. Holland

Project Team: John F. Doyle James M. Eyer Franjee Hay Ralph H. Hollenbacher Jennifer A. Martin Susan K. Moxley.

Prepared for:

Pacliflc Gas and Electric Company Department of Research and Development 3400 Crow Canyon Road San Ramon, Californla 94583

(C) 1993 by PG\&E 


\section{DISCLAIMER}

This report was prepared as an account of work sponsored by an agency of the United States Government. Neither the United States Government nor any agency Thereof, nor any of their employees, makes any warranty, express or implied, or assumes any legal liability or responsibility for the accuracy, completeness, or usefulness of any information, apparatus, product, or process disclosed, or represents that its use would not infringe privately owned rights. Reference herein to any specific commercial product, process, or service by trade name, trademark, manufacturer, or otherwise does not necessarily constitute or imply its endorsement, recommendation, or favoring by the United States Government or any agency thereof. The views and opinions of authors expressed herein do not necessarily state or reflect those of the United States Government or any agency thereof. 


\section{DISCLAIMER}

Portions of this document may be illegible in electronic image products. Images are produced from the best available original document. 
Report Issued:

January 8, 1993

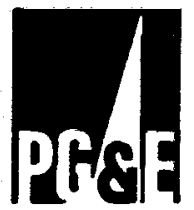

\section{Research and \\ Development}

Summary of New Generation

Technologies and Resources

Advanced Energy Systems

Report 007.6-92.3

Program Manager: George A. Hay III

Technology Ássessment, Monftoring, and Scanning (TAMS)

Final Report, December 1992

Project Managers: David W. Behrens Donna S. Lindquist

Ronnie A. Holland

Project Team: John F. Doyle James M. Eyer

Franjee Hay

Ralph H. Hollenbacher

Jennifer A. Martin

Susan K. Moxley

Prepared for:

Pacific Gas and Electric Company

Department of Research and Development

3400 Crow Canyon Road

San Ramon, Californla 94583

C 1993 by PG\&E 


\section{LEGAL NOTICE}

Pacific Gas and Electric Company (PG\&E) makes no warranty or representation, expressed or implied, with respect to the accuracy, completeness, or usefulness of the information contained in this report, or that the use of any information, apparatus, method, or process disclosed in this report may not infringe upon privately owned rights. Nor does PG\&E assume any liability with respect to use of, or damages resulting from the use of, any information, apparatus, method, or process disclosed in this report.

\section{1993 by PG\&E}

All Rights Reserved 


\section{CONTENTS}

Page

Program Perspective $x i$

Acknowledgments xvii

Glossary xix

\section{COST AND PERFORMANCE DATABASE}

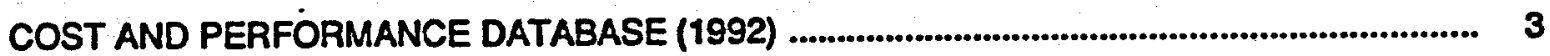

\section{RENEWABLES}

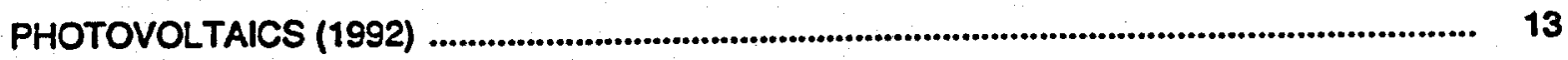

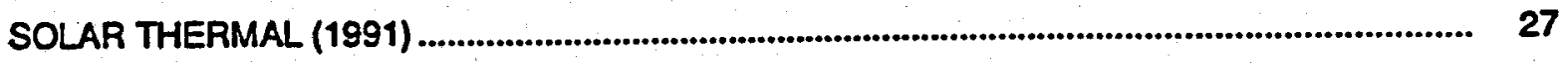

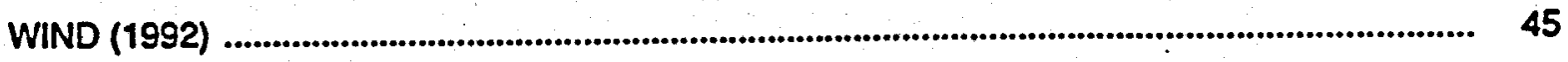

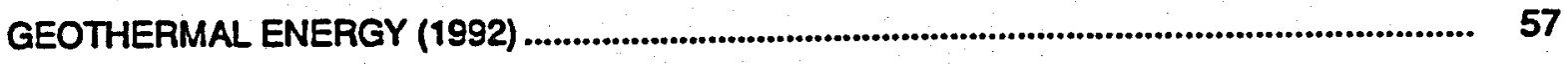

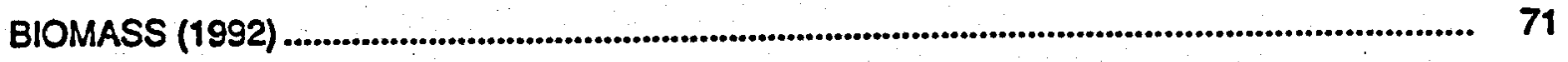

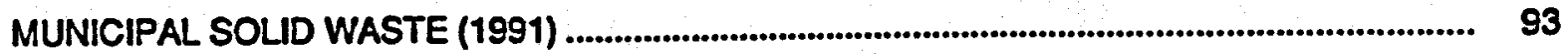

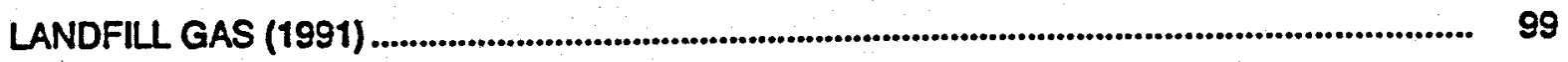

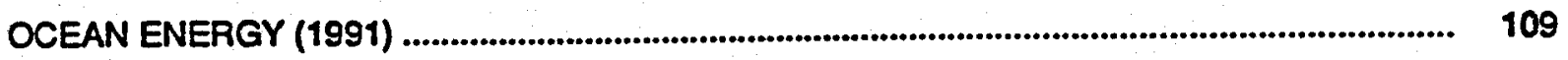

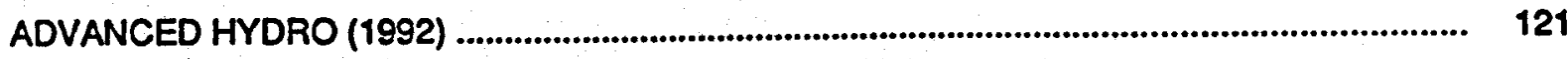

\section{ENERGY STORAGE}

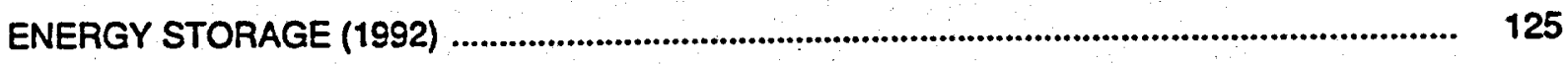

\section{ADVANCED FOSSIL CONVERSION}

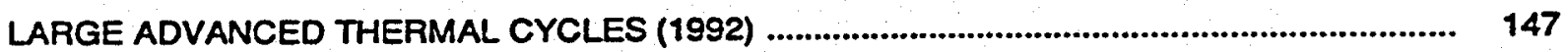

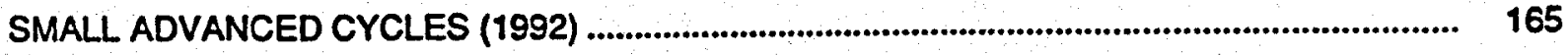

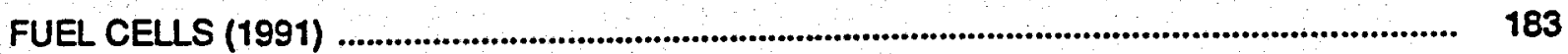

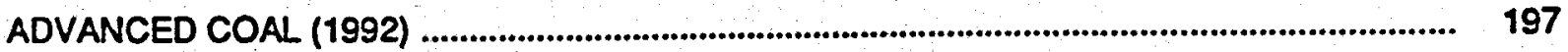

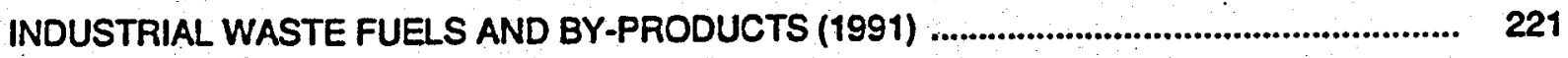

\section{ADVANCED NUCLEAR POWER}

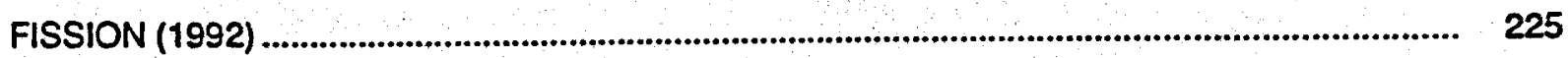

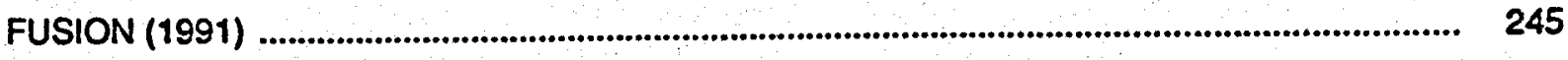

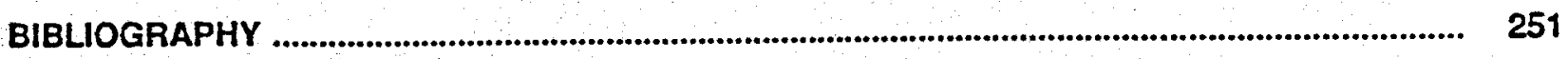




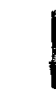




\section{FIGURES}

PHOTOVOLTAICS

1 PV module costs and sales (historic and torecast) ..................................................... 21

2 Diffusion model of PV utility applications .................................................................................. 24

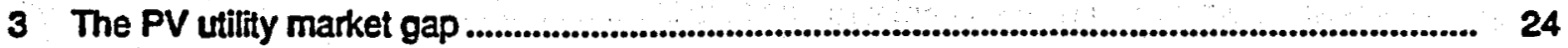

\section{SOLAR THERMAL}

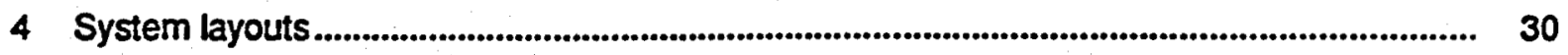

5 Existing and planned deployment capacities ............................................................. 33

6 Comparison of solar central receiver and combined cycle generation costs ...................... 40

WIND

7 Worldwide wind energy resource distribution estimates ............................................... 48

8 Wind turbine configurations ..................................................................................................... 49

9 Five areas of extensive wind development in Califomia .............................................. 51

\section{BIOMASS}

10 Schematic of atmospheric fluidized bed combustor ..................................................... 76

11 Schematic of spreader stoker fired steam generator ............................................................ 77

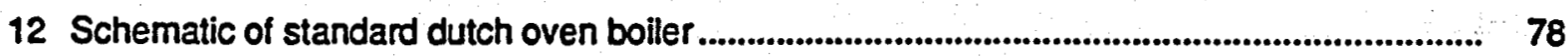

13 Califormia biomass fuels demand: new capacity/total demand during the 1980 s ................. 83

14 Environmental considerations associated with biomass use ......................................... 86

\section{LANDFILL GAS}

15 Schematic of gas recovery system ............................................................................... 102

16a High-Btu process-adsorption-compression membrane separation diagram ......................... 105

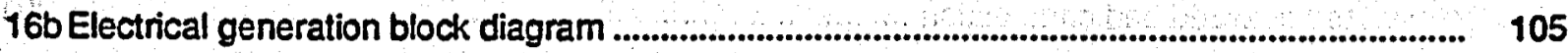

\section{OCEAN ENERGY}

17 Projected capital investment for a 27-MWe heaving buoy wave energy plant........................ 111

18 Sensitivity analysis of capltal Investment for a 27-MWe heaving buoy wave energy plant.... 112

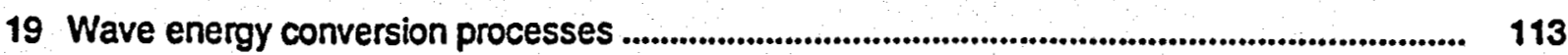

20 Resource supply curve for northern Califomia ................................................................... 117 


\section{FIGURES (Cont.)}

Page

\section{ENERGY STORAGE}

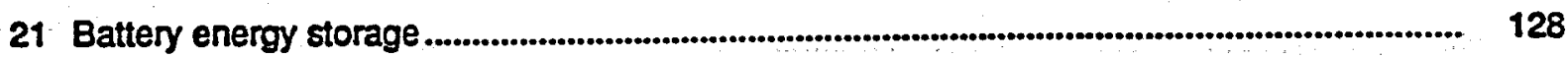

22 Schematic of a CAES plant using a depleted gas field ........................................................ 129

23 Superconducting magnetic energy storage ...................................................................... 130

24 Helms 1,000-MW pumped hydroelectric energy storage .................................................... 131

\section{LARGE ADVANCED THERMAL CYCLES}

25 Projected performance improvements in advanced natural gas converrsion technologies .... 149

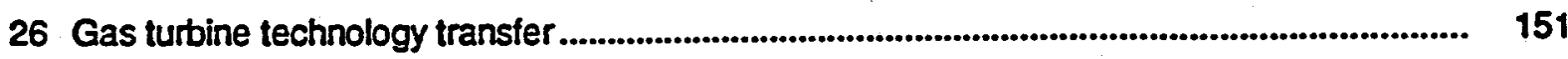

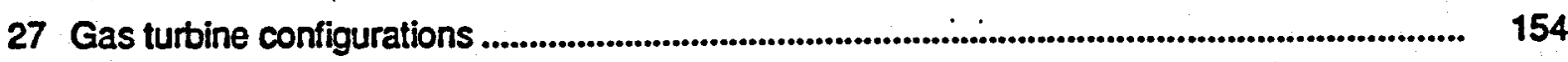

28 Carbon dioxide emissions for natural-gas-fueled technologies ....................................... 161

\section{SMALL ADVANCED CYCLES}

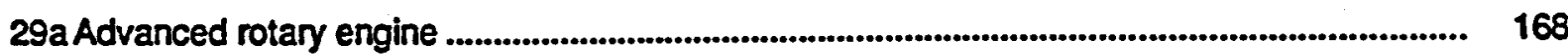

29b Advanced gas turbine (steam-injected cycle) ....................................................................... 168

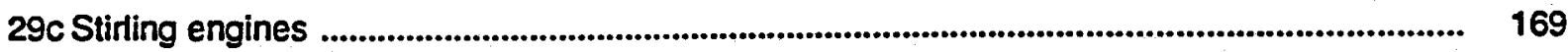

\section{FUEL CELLS}

30 Schematic representation of a typical tuel cell .................................................................... 186

31 Economic comparison: mature MCFC vs. combined cycle alternative .................................... 194

\section{ADVANCED COAL}

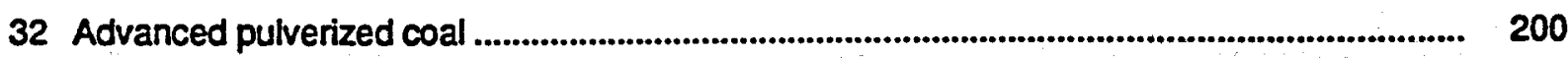

33 Atmospheric fluidized bed combustion ......................................................................... 200

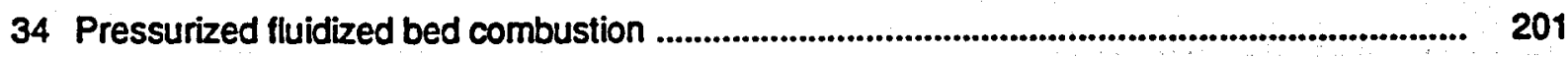

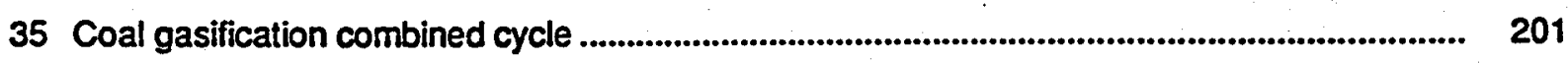

36 Traditional utility view of year 2005 electric generating resources .......................................... 214

37 A multi-attribute view of year 2005 electric generating resources ......................................... 218

38 Year 2015 repowering scenarios ...................................................................................... 219 


\section{FIGURES (Cont.)}

\section{FISSION}

Page

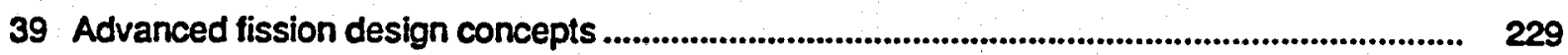

40 Traditional utility view of year 2005 electric generating resources........................................ 240

41 A multi-attribute view of year 2005 electric generating resources ...................................... 242 


$$
\text { , }
$$




\section{TABLES}

\section{Page}

COST AND PERFORMANCE DATABASE

1 Advanced Gas/Oil Baseload Generation Options ............................................................... 4

2 Advanced Gas Intermediate Load Generation Options ................................................... 5

3. Advanced Gas Distributed Generation Options .............................................................. 6

4 Peak Load Generation and Storage Options ....................................................................... 7

5 Renewable Technologies, Baseload Generation Options ............................................... 8

6 Renewable and Storage Technologies, Intermediate Load Generation Options .................. 9

7 Advanced Coal and Nuclear, Baseload Central Station Options ....................................... 10

\section{PHOTOVOLTAICS}

8 Photovoltaic Plant Characteristics and Costs ........................................................... 14

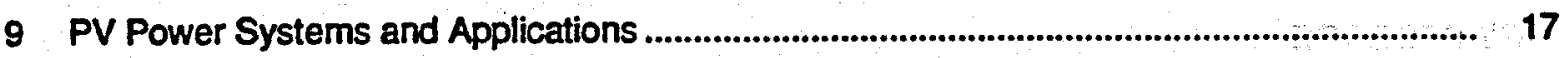

10 Photovoltaic Cell Technologies .................................................................................... 20

\section{SOLAR THERMAL}

11 Solar Themal Plant Characteristics …....................................................................... 28

WIND

12 Wind Power Plant Characteristics ............................................................................ 46

\section{GEOTHERMAL ENERGY}

13 Capital Costs for Recently Constructed Geothermal Plants .......................................... 58

14 Hydrothermal Resources Within PG\&E's Service Area ............................................... 62

15 Hydrothermal Resources in the Salton Trough, California......................................... 63

16 Hydrothermal Resources in the Basin and Range, Oregon Plateaus, Snake River Plain and Northem Rockies Provinces ...................................................................... 64

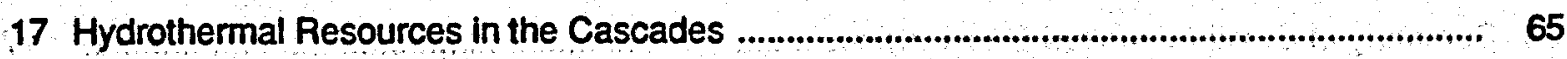




\section{TABLES (Cont.)}

BIOMASS

18 Conversion of Biomass into Electricity in $\mathbf{1 9 9 0}$ 74

19 Comparison of Current Biomass Combustion Technology Emissions Guarantees 79

20 Biomass Plants Currently Serving PG\&E's Grid 80

21 Available Waste and Residue Fuels for the Califomia Biomass Energy Industry 84

22 Comparison of Wood Buming Technologies 89

\section{OCEAN ENERGY}

23 Cost and Performance Data for Planned or Hypothetical Wave Power Plants

24 Wave Energy Facilities

\section{ENERGY STORAGE}

25 Energy Storage Characteristics

26 Advanced Energy Storage Projects (Current and Planned) 133

27 Cost and Pertormance of Battery Energy Storage Technologies 135

28 Cost and Pertormance of CAES, SMES, and Pumped Hydro Energy Storage Technologies. 136

29 Cost and Performance of Flywheel Technology 137

\section{LARGE ADVANCED THERMAL CYCLES}

30 Projected Improvements in Large Advanced Thermal Cycle Technologies 148

31 Emerging Aeroderivative Gas Turbine Engines for Power Generation Applications

\section{SMALL ADVANCED CYCLES}

32 Typical Applications for Small Advanced Cycles

33 Summary of Small Advanced Reciprocating and Rotary Engines

34 Summary of Small Advanced Gas Turbines 175

35 Summary of Stirling Engines. 177

36 . Fuels for Small Advanced Cycles 


\section{TABLES (Cont.)}

\section{Page}

\section{FUEL CELLS}

37 Fuel Cell Development History and Cost Projections ........................................................... 184

38 Applications for Fuel Cells ................................................................................................... 187

39 Fuel Cell Emission Levels ................................................................................................... 190

40 Technical and Economic Characteristics of Competing Technologies .................................... 193

\section{ADVANCED COAL}

41 AFBC Projects in Califomia Fueled by Coal and Petroleum Coke ....................................... 203

42 Status of Advanced Coal Technologies ................................................................................ 207

43 Economic Comparison Between Advanced Nuclear, Coal, and Natural Gas New Generation Options

44 Estimated Economics for Advanced Coal Conversion Technologies for Electric Power Generation

\section{FISSION}

45 U.S. Advanced Fission Power Plant Features ...................................................................... 228

46 Economic Comparison Between Advanced Nuclear, Coal, and Natural Gas New Generation Options 


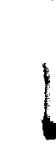




\section{PROGRAM PERSPECTIVE}

The mission of the PG\&E R\&D program is to contribute to the company's competitive advantage by managing technology change and reducing technology risk. The formula for accomplishing this mission, stated very simply, is to "seek, select, try, and apply." Figure PP-1 illustrates this concept of R\&D management.

In the strategic research ("seek") phase, R\&D's task is to identify technologies which may be of value to the company, gatherbusiness intelligence, and provide information on emerging technologies and new applications of existing technologies. This can be approached informally or, as in the case of the technology assessments summarized here, in a more systematic way.

In 1989, the Advanced Energy Systems (AES) Technology Information Module (TIM) project began preparing a systematic compilation of information about emerging electric generation technologies, and has since published a series of 17 TIM reports, as well as a cost and performance database, which distills basic quantitative information on the various technologies of interest and presents the information in a standard format. The objective of the TIMs is to enhance and document the PG\&E R\&D Program's understanding of the technology status, resource potential, deployment hurdles, commercial timing, PG\&E applications and impacts, and R\&D issues of advanced technologies for electric utility applications in Northem California.

The project addressed two basic needs not met by technology assessments available from other sources: it evaluated each emerging option in relation to the opportunities, resources, and constraints unique to PG\&E; and it provided objectivity and balance. In general, the plethora of other assessments available fall short of these needs because they are not complete, providing generic information of interest to the technology developer rather than specific information of interest to the technology user. Also, typically, for emerging options, costs are understated and performance overstated to widely varying degrees, depending on the maturity and objectivity of the evaluator.

To assure the needed degree of completeness and accuracy, a standard categorization of TIM information was established, and a review process was completed that engaged the knowledge and insight of PG\&E in-house experts. Each TIM typically includes the following categories of intormation:

- Technology description

- PG\&E applications

- Development and commercialization status

- Fuels and energy resource assessment 


\section{MISSION STATEMENT}

The mission of R\&D is to contribute to the company's competitive advantage by managing technology change and reducing technology risk. To accomplish this R\&D will:

\section{SEEK}

Identify technologies which may be of value to the company, gather business intelligence and provide information on emerging technologies and new applications of existing technologies.

\section{SELECT}

Evaluate and select emerging technologies and new applications of existing technologies to be developed and applied by the company.

\section{TRY \& APPLY}

Catalyze the rapid application and implementation of technology by the company through demonstrations, technology transfer and effective communication.

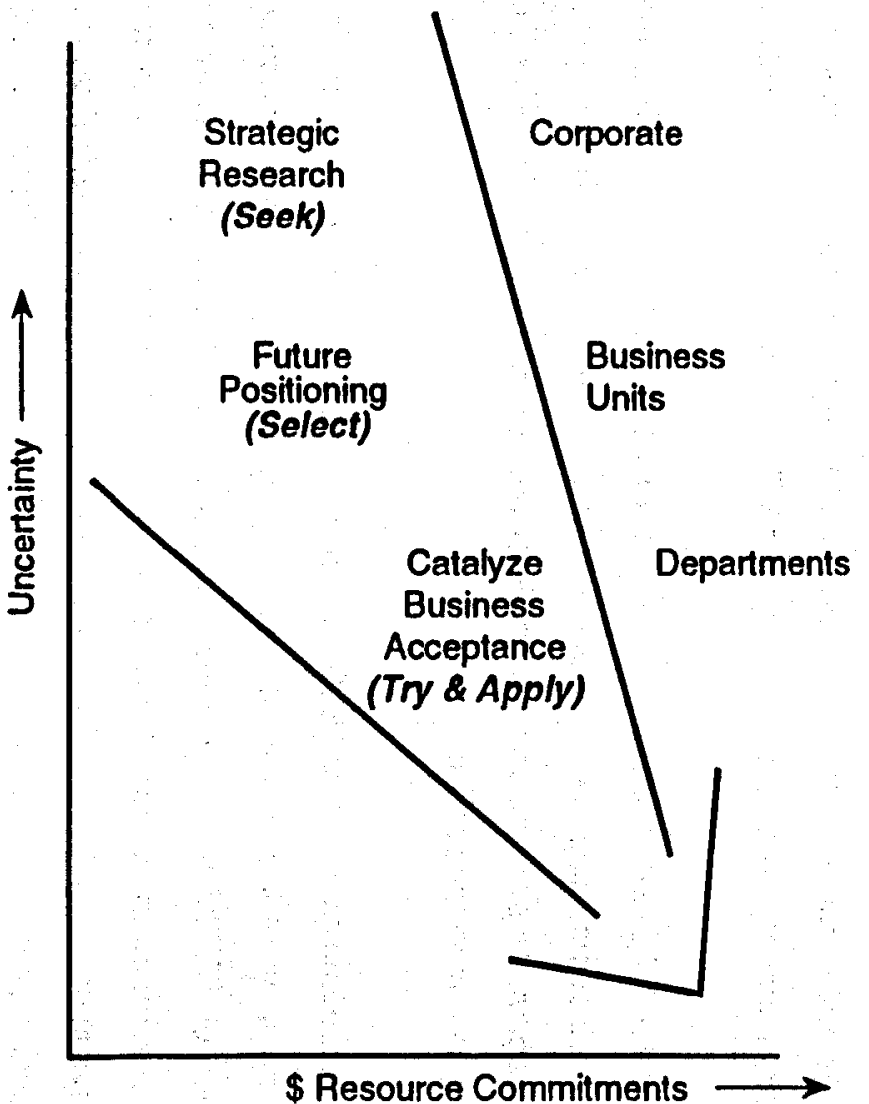

\$ Resource Commitments

Figure PP-1. Research \& Development Program. 
- Environmental impacts

- Deployment considerations and hurdles

- Technology trends and forecasts

- PG\&E and utility industry impacts and implications

- R\&D needs and opportunities

This compendium includes a program perspective on the project, a glossary, a summary of each TIM, updated information on the status and trends of each technology, and a bibliography.

As might be expected, the goals of completeness and accuracy were attained to varying degrees, depending on the quality of contractor work products and the diligence of in-house reviewers. Further, and more importantly, none of the technologies assessed in the TIM project is standing still. Those still under development, and especially those evolving in the market as a result of experience-driven innovation, are truly moving targets. The reader is strongly encouraged to regard the information presented in the database and TIM modules as having a finite "sheff life" which may vary significantly depending on the technology and type of information. The information may be regarded as up-to-date as of 1991, since most of the report draftswere in final form prior to 1992.

The information in the TIMs is consistent with the choice of emerging options targeted ("selected") for accelerated development by the AES R\&D program. Moving into the "try and apply" phase required careful selection of technologies and projects. PG\&E must concentrate its resources and efforts if it is to have the desired impact-accelerating the technical and economic readiness of currently unavailable technologies. The selection criteria were:

1. The technology is environmentally preferred.

2. The technology uses resources which are abundant and indigenous to Northern California or abundant and accessible via PG\&E electric and natural gas transmission systems.

3. It has reasonable probability of cost-effectiveness in 2000-2010 without ratepayer subsidy.

4. It requires PG\&E initiative to capture acceleration potential.

5. The California Energy Commission (CEC) targeted it as an "opportunity technology."

The technologies that met these criteria are identified in Figure PP-2. PG\&E "try and apply" activity is justified in each case for the following reasons:

- Wind will be a least-cost supply option, but resources in northerncentral California are limited. No other utility or national program is addressing the need to get the maximum amount of economy energy from a limited wind resource. 


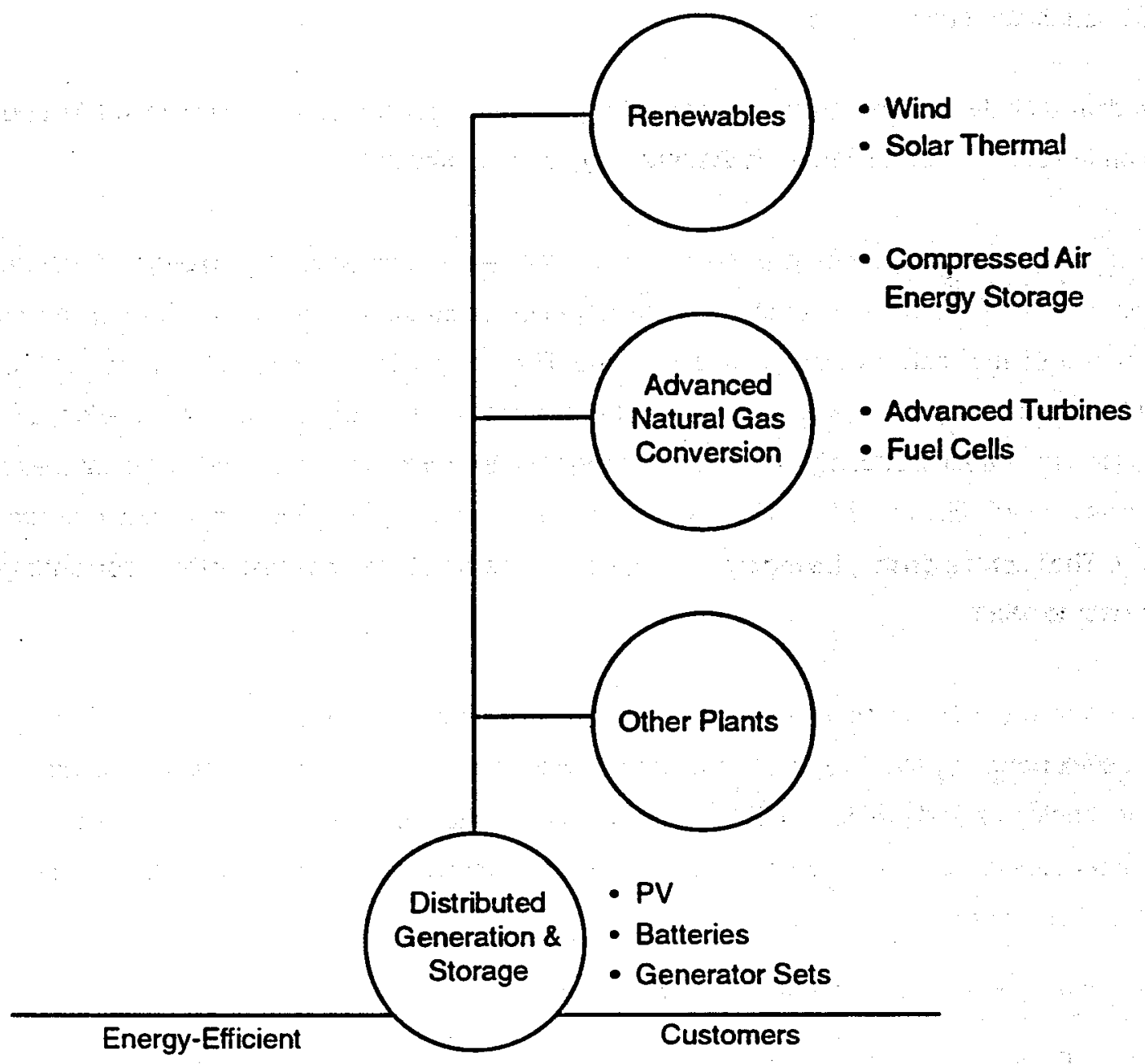

Figure PP-2. Target technologies meet selection criteria. 
- Commercial solar thermal technology is stalled as a result of LUZ's bankruptcy. The U.S. national program is focused on altematives to the LUZ approach. These altematives have little if any hope of meeting PG\&E's selection criteria. PG\&E is the only U.S. utility addressing the need to revive and improve the only commercial solar bulk power technology.

- Compressed air energy storage resources in the San Joaquin Valley are outstanding, but improving the understanding of underground reservoir development and performance issues is the key to managing technical risk. No otherutilities or programs will be dealing with exactly the same geology or plant optimization boundary conditions that apply to PG\&E. Therefore, no successful development and application will occur in Northern California unless PG\&E does its technical homework.

- Advanced aeroderivative gas turbine applications are not unique to PG\&E, but leadership of utility efforts to adapt aircraft turbines to the electric power market is. PG\&E's commercially successful effort with GE in developing tts LM5000 steam-injected gas turbine (STIG) product, PG\&E's subsequent intercooled steam-injected gas turbine (ISTIG) development initiative, the CEC's intense interest, and the California utilities' lange share of the nation's natural-gas-based generation-all argue for PG\&E to take the lead in catalyzing accelerated development of advanced turbine concepts.

- Emerging solar photovoltaic and fuel cell technologies have also captured the imagination and Interest of many utilities. Multiple fuel cell and solar photovoltaic products will be introduced to the electric power market over the next decade. If successful, these products could accelerate the evolution of the regional electric supply infrastructure toward the "distributed utility" model championed by R\&D. PG\&E has more firsthand experience with solar photovoltaics and carbonate fuel cell systems than any utility in the United States, operates world-class test facilities in both technologies, and recelves royalties on the intemal reforming carbonate fuel cell innovation it sponsored in the mid-1980s. Continuation of costshared test activity will position PG\&E to make accurate and timely assessments of technology readiness, market timing, and business opportunities.

- Other distributedgeneration and storage technologies such as batteries and modularnatural gas engine-based generation offer a shorter term potential to improve electric and gas distribution system asset utilization and improve PG\&E's ability to offer competitive prices to customers. Regrettably, the Electric Power Research Institute (EPRI), the Gas Research Institute (GRI), the U.S. Department of Energy (DOE), and traditional utility equipment vendors have yet to formulate focused programs that are responsive to the market need and opportunity, so it is in PG\&E's interest to be more proactive than might othenwise be required.

Certainly, these choices will have to be continually revalidated as development and demonstration proceeds. Within each target technology category, a range of basic system configuration and component choices must be made (e.g., the choice of carbonate fuel cell technology within the fuel cell category, the choice of a oncethrough internal reforming system configuration, and the choice of a bumer vs. a catalytic conversion device for heating inlet air with unconverted fuel). Thus, ongoing technology assessment activity will continue as a key part of the AES program, providing R\&D program managers and their business unit clients with a radar screen showing the location, speed, and direction of emerging generation options. 


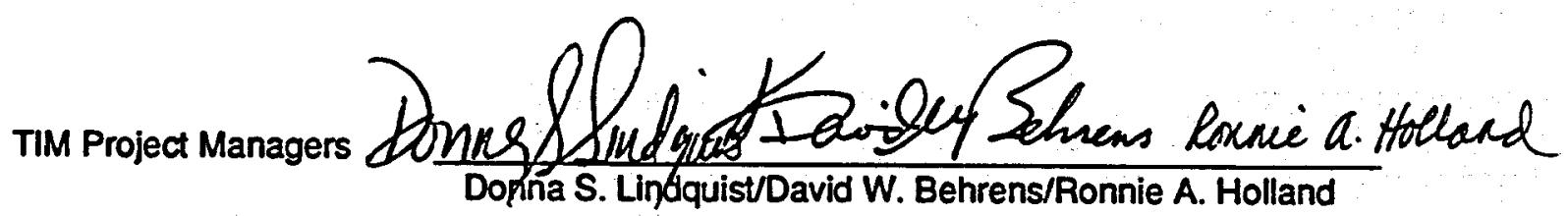

TIM Program Manager

Research Director

Advanced Energy Systems
Shore A. Aton III

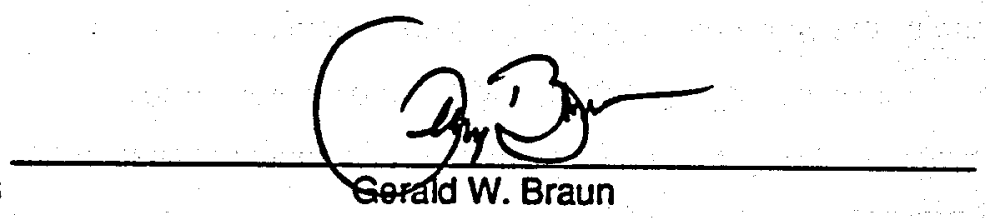

0076923/60008339/dp70

xvi 


\section{ACIKNOWLEDGMENTS}

The completion of a broad-ranging effort like the Technology Information Module Project required the cooperation and perseverance over several years by many people. In addition to the authors, contributors, and reviewers listed below, special thanks go to AES Director Gerry Braun; Program Manager George Hay; Project Managers Dave Behrens, Donna Lindquist, and Ronnie Holland; and project teammembers including John Doyle, Jim Eyer, Franjee Hay, Ralph Hollenbacher, Jennifer Martin, and Susan Moxley. The support services provided by the Desktop Publishing and Graphics groups helped finalize the production and assembly of text and ant, and we thank these individuals: John Buchholz, Laraine Chaney, Carol Crews, Shirley Dexter, Sheila Doherty, Mel Douglas, Lauren Eastman, Sue K. Gubb, Mickey Hops, Judie Hurtz, Gema Lopez, Roslyn Raley, and Donna Smailes.

\section{CONSULTANTS}

AeroVironment Inc.

Bechtel Group, Inc. (Nuclear Power Systems, R\&D)

Kay Firor (Blue Mountain Energy)

Ralph H. Hollenbacher (Energy Options)

John F. Doyle (Engineering Perspectives, Inc.)

Future Resources Associates, Inc.

Charles Greene Consultants

International Engineering Services Corporation

Meridian Corporation

EA Mueller, Inc.

George Wiltsee (NEOS Corporation)

Melissa M. Reading, Ph.D.

Dr. Susan Schoenung and Dr. Wayne Meier

(W. J. Schafer Associates, inc.)

Science Applications InternationalCorporation (SAIC)

SEASUN Power Systems

D.J. Wilhelm, D.R. Simbeck, S. Vejtasa,

R.L. Dickenson (SFA Pacific, Inc.)

TENERA Environmental Services

\section{CONTRIBUTORS/REVIEWERS}

\author{
Ken Abreu \\ Clint Astworth \\ Jonne Berning \\ Max Blanchet \\ Gerald W. Braun \\ Sally M. C. de Becker \\ Ray J. Dracker \\ EDAW Inc. \\ Roberto Gutierrez \\ Robert L. Hammond \\ Douglas J. Herman \\ Tom Hillesland \\ Joseph J. Iannucci \\ Mary A. Ilyin \\ Christina Jennings \\ James A. Kimball \\ Paul Langholz \\ Thomas A. Lott \\ Jo L. Meglen \\ Karen Morris \\ Dan Quesenberry \\ Jay Raggio \\ Robert B. Schainker (EPRI) \\ Daniel S. Shugar \\ Don R. Smith \\ William J. Steeley \\ Jane H. Tumbull \\ Howard J. Wenger \\ Charles 0 . White \\ Rick O. Winter
}




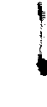




\section{GLOSSARY}

ANSI

API

Baseload

BLM

BOS

BPA

CAES

Capacity

Capacity Factor

CEC

Cogeneration

CPUC

Distributed Generation.

DOE

EPRI

FERC

GRI

HHV

Hybrid Systems

IEA
American National Standards Institute

American Petroleum Institute

Minimum load demanded over time on a power company's generation system.

Bureau of Land Management

Balance of system

Bonneville Power Administration

Compressed air energy storage. Air is compressed using off-peak electric energy and stored underground for later use in an expansion turbine.

Amount of electric power for which a generating unit, generating station, or other electrical apparatus is rated either by the user or manufacturer.

Ratio of the average operating load of an electric power generating unit for a period of time to the capacity rating of the unit during that period.

California Energy Commission

Production of thermal energy and electrical or mechanical powerf from the same fuel source.

California Public Utility Commission

characteristics match local electricity, economic, and environmental demands.

Department of Energy

Electric Power Research Institute

Federal Energy Regulatory Commission

Gas Research Institute

Higher heating value. Any fuel containing hydrogen yields water as one product of combustion. High heat value is calculated assuming all watervaporcondensed. The HHV efficiency for natural gas conversion is about $10 \%$ lower than Low Heat Value (LHV) (see below).

Combinations of generators, non-dispatchable technologies such as wind or photovoltaics, and storage.

Intemational Energy Agency 
IPP

LHV

NOI

NREL

Nuclear

O\&M

Peak Load

PURPA

QF

Retail Wheeling

SCE

SDG\&E

SMUD

SNLA

Wholesale Wheeling
Independent Power Producers. A private entity building new generation facilities to sell in wholesale (i.e., bulk power) markets.

Lower heating value. Any fuel containing hydrogen yields water as one product of combustion. Low heat value is evaluated assuming no water vapor condensed. In Germany, the low heat value of the fuel is used in calculating efficiencies of internalcombustion engines. In the United States, the high value is specified by the American Society of Mechanical Engineers (ASME) Power Test Codes (see HHV).

Notice of Intent

National Renewable Energy Lab (formerly: Solar Energy Research Institute (SERI)

As used in this report, nuclear refers to fission and fusion energy technologies.

Operation and maintenance

Maximum demand for electric power that determines the generating capacity required by the utility.

Public Utility Regulatory Policies Act of 1978. The act required utilities to purchase power and provide back-up services to cogenerators and certain small power production facilities (qualifying facilities) that made use of renewable energy sources and various waste fuels.

Qualifying facility (under PURPA). A small power producer or cogenerator that can sell its electricity to public utilities.

The use of a transmission system by a utility or an independent power producer to send power directly to a retail customer (see Wholesale Wheeling).

Southem California Edison

San Diego Gas and Electric

Sacramento Municipal Utility District

Sandia National Laboratories

The use of a transmission system by a utility or an independent power producer to send electricity to a wholesale customer, usually another utility. 



\section{COST AND PERFORMANCE DATABASE}

The PG\&E Advanced Energy Systems (AES) R\&D Program funds over $\$ 5$ million annually to investigate future electric supply options in California. Information on the cost and performance of future PG\&E electric supply options is a major product of these investigations. The intent of the AES Cost and Performance Database was to compile the most recent information available from the program for R\&D and business unit users of information on new generation technologies. The database evolved out of the R\&D 1988 Scenario Evaluation and R\&D Choices (SEARCH) Study, Phase 1,1 and it drew extensively on the 17 Technology Information Module (TIM) reports, which attempt to document qualitative as well as quantitative information. Although no future updates are planned, the database is also intended to serve as a repository for new information as it becomes available and as a reference for evaluating the significance of new information.

Tables 1 through 7 summarize the results of the cost and performance study for each technology case. Summary Tables 1 through 7 are:

1. Advanced Gas/Oil Baseload Generation Options

2. Advanced Gas Intermediate Load Generation Options

3. Advanced Gas Distributed Generation Options

4. Peak Load Generation and Storage Options

5. Renewable Technologies Baseload Generation Options

6. Renewable and Storage Technologies Intermediate/Peak Load Generation Options

7. Advanced Coal and Nuclear Baseload Central Station Options

This database is being incorporated into a sottware database management program ("E-TRENDS"), which can extract relevant information and perform financial and economic analyses.

1J.F. Doyle, J. M. Eyer, and G. A. Hay III, Scenario Evaluation and Research Choices (SEARCH) Study (San Ramon, Califomia: PG\&E, Report 005.88-1, 1988).

$0076923 / 60008339 / \mathrm{dp} 70$ 
Table 1

Advanced Gas/Oil Baseload Generation Options

(1990 Dollars)

$A$

\begin{tabular}{|c|c|c|c|c|c|c|c|c|c|c|c|c|}
\hline $\begin{array}{c}\text { TABLE } \\
\text { NO. }\end{array}$ & $\begin{array}{l}\text { TECHNOLOGY CASE NAME } \\
\text { (Refer Appendix D Tables "BG1 thru BG9" } \\
\text { For Detailed Data Sheets) }\end{array}$ & $\begin{array}{c}\text { Commercial } \\
\text { Operation } \\
\text { Time Frame } \\
\text { (Note 1) }\end{array}$ & $\begin{array}{c}\text { Unit } \\
\text { Size } \\
\text { (MWe) }\end{array}$ & $\begin{array}{l}\text { Plant } \\
\text { Size } \\
\text { No of } \\
\text { Units }\end{array}$ & $\begin{array}{l}\text { Total } \\
\text { Cost } \\
\text { (Not } \\
\text { (Low) }\end{array}$ & $\begin{array}{l}\text { Plant } \\
(\$ / k W) \\
\text { to } 3) \\
\text { (Ren }\end{array}$ & $\begin{array}{l} \\
\text { (High) } \\
\end{array}$ & $\begin{array}{c}\text { O\&M } \\
\text { Fixed } \\
(\$ / \mathrm{KW}-\mathrm{Y})\end{array}$ & $\begin{array}{c}\text { O\&M } \\
\text { Varlable } \\
\text { (Milis/kWh) }\end{array}$ & $\begin{array}{l}\text { Full Load } \\
\text { Hoat Plate } \\
\text { HHW } \\
\text { (Btu/kWh) }\end{array}$ & $\begin{array}{l}\text { Efficlency } \\
\text { (HHV-\%) }\end{array}$ & $\begin{array}{l}\text { Quality of } \\
\text { Data (Scale } \\
\text { (1)-Bost To } \\
\text { (5)-Worst }\end{array}$ \\
\hline $\begin{array}{l}\text { BG1 } \\
\text { BG1a } \\
\text { BG1a }\end{array}$ & $\begin{array}{l}\text { Combined Cycle (one Frame 7E;one steam turb) } \\
\text { Combined Cycle (one Frame 7F;one steam turb) } \\
\text { Combined Cycle (one Frame 7F;one steam turb) }\end{array}$ & $\begin{array}{l}\text { Near } \\
\text { Near } \\
\text { Long }\end{array}$ & $\begin{array}{l}123 \\
222 \\
235\end{array}$ & $\begin{array}{l}4 \\
2 \\
2\end{array}$ & $\begin{array}{l}500 \\
475 \\
475\end{array}$ & $\begin{array}{l}600 \\
575 \\
575\end{array}$ & $\begin{array}{l}700 \\
650 \\
650\end{array}$ & $\begin{array}{l}9 \\
9 \\
9\end{array}$ & \begin{tabular}{l|}
3 \\
3 \\
3
\end{tabular} & $\begin{array}{l}7887 \\
7400 \\
7100\end{array}$ & $\begin{array}{l}43.4 \\
46.1 \\
48.1\end{array}$ & $\begin{array}{l}3 \\
3 \\
3\end{array}$ \\
\hline$\overline{B G 2}$ & PQ\&E IDA Combined Cycle (Fr 7F-Dry Cooling) & Near & 217.3 & 1 & 600 & 600 & 600 & 30 & 0.9 & 7686 & 44.4 & 2 \\
\hline $\begin{array}{l}\text { BG3 } \\
\text { BG3a } \\
\text { BG3b }\end{array}$ & $\begin{array}{l}\text { Current Aeroderivative (eg LM6000 Combined Cycie) } \\
\text { LM6000 Com Cyc(two } 42 \text { MW GT;one 25MW steam) } \\
\text { Advanced Aeroderivative (See Note 2) }\end{array}$ & $\begin{array}{l}\text { Near } \\
\text { Near } \\
\text { Long }\end{array}$ & \begin{tabular}{r|}
50 \\
109 \\
160
\end{tabular} & \begin{tabular}{l|l}
4 & \\
2 & \\
1 &
\end{tabular} & $\begin{array}{l}600 \\
500 \\
600\end{array}$ & $\begin{array}{l}700 \\
600 \\
600\end{array}$ & $\begin{array}{l}800 \\
700 \\
800\end{array}$ & \begin{tabular}{l|}
9 \\
9 \\
9
\end{tabular} & $\begin{array}{l}7 \\
3 \\
7\end{array}$ & $\begin{array}{r}7760 \\
7650 \\
6100-6700\end{array}$ & $\begin{array}{r}44.0 \\
44.6 \\
51.56 \%\end{array}$ & $\begin{array}{l}3 \\
3 \\
5\end{array}$ \\
\hline $\begin{array}{l}\text { BG3c } \\
\text { Ba3d }\end{array}$ & $\begin{array}{l}\text { LM6000 Simple Cycle } \\
\text { Advanced Simple Cycle LM6000 (intercooled) }\end{array}$ & $\begin{array}{l}\text { Near } \\
\text { Long }\end{array}$ & \begin{tabular}{l|}
40 \\
80
\end{tabular} & $\begin{array}{l}4 \\
2\end{array}$ & $\begin{array}{l}450 \\
400\end{array}$ & $\begin{array}{l}500 \\
450\end{array}$ & $\begin{array}{l}600 \\
550\end{array}$ & $\begin{array}{l}9 \\
9\end{array}$ & \begin{tabular}{l|}
7 \\
7
\end{tabular} & $\begin{array}{l}9230 \\
8754\end{array}$ & $\begin{array}{r}37 \\
39.0\end{array}$ & $\begin{array}{l}3 \\
\end{array}$ \\
\hline $\begin{array}{l}\text { BG4 } \\
\text { BG4 }\end{array}$ & $\begin{array}{l}\text { Thermally Enhanced Oll Recovery (Frame 7E) } \\
\text { Thermally Enhanced Oll Rocovery (Frame 7F) }\end{array}$ & $\begin{array}{l}\text { Near } \\
\text { Long }\end{array}$ & $\begin{array}{r}84 \\
150\end{array}$ & $\begin{array}{l}4 \\
2\end{array}$ & $\begin{array}{l}600 \\
600\end{array}$ & $\begin{array}{l}660 \\
660\end{array}$ & $\begin{array}{l}720 \\
720\end{array}$ & $\begin{array}{l}9 \\
9\end{array}$ & 3 & $\begin{array}{l}11740 \\
10964\end{array}$ & $\begin{array}{l}29.1 \\
31.1\end{array}$ & $\begin{array}{l}3 \\
4\end{array}$ \\
\hline BG5 & $\begin{array}{l}\text { Repowering Ges Turbine Topping Cycle } \\
\text { (See Note } 5 \text { ) }\end{array}$ & Near & 215 & 1 & 800 & 1000 & 1500 & 8 & 6 & 8000 & 42.7 & $\overline{2}$ \\
\hline $\begin{array}{l}\text { BG6 } \\
\text { BQ6 }\end{array}$ & $\begin{array}{l}\text { Phosphoric Acid Fuel Coll } \\
\text { Phosphorle Acld Fuel Cell }\end{array}$ & $\begin{array}{l}\text { Near } \\
\text { Long }\end{array}$ & \begin{tabular}{l|}
10 \\
25
\end{tabular} & $\begin{array}{l}1 \\
4\end{array}$ & $\begin{array}{r}1400 \\
900\end{array}$ & $\begin{array}{l}1500 \\
1000\end{array}$ & $\begin{array}{l}1900 \\
1200\end{array}$ & $\begin{array}{l}9 \\
8\end{array}$ & \begin{tabular}{r|}
6.5 \\
5
\end{tabular} & $\begin{array}{l}8300 \\
7000\end{array}$ & $\begin{array}{l}41.1 \\
48.8\end{array}$ & $\begin{array}{l}3 \\
4\end{array}$ \\
\hline $\begin{array}{l}\text { BG7 } \\
\text { BG7 }\end{array}$ & $\begin{array}{l}\text { Molten Carbonate Fuel Cell } \\
\text { MCFC (20 MW steam turb bottoming cycle) }\end{array}$ & $\begin{array}{l}\text { Near } \\
\text { Long }\end{array}$ & \begin{tabular}{r|}
2 \\
100
\end{tabular} & $\begin{array}{l}1 \\
1\end{array}$ & $\begin{array}{r}1500 \\
800\end{array}$ & $\begin{array}{l}2000 \\
1000\end{array}$ & $\begin{array}{l}2500 \\
1500\end{array}$ & $\begin{array}{l}7 \\
6\end{array}$ & $\begin{array}{r}10 \\
9\end{array}$ & $\begin{array}{l}6825 \\
5700\end{array}$ & $\begin{array}{l}50.0 \\
59.9\end{array}$ & 4 \\
\hline BG8 & $\begin{array}{l}\text { Solid Oxide Fuel Cell } \\
\text { (16.2 MW Fuel Cell;3.8 MW Steam Turbine) }\end{array}$ & Long & 20 & 1 & 1000 & 1450 & 1750 & 8 & 11 & 6200 & 55.1 & 5 \\
\hline$\overline{B G 9}$ & $\begin{array}{l}\text { Solid Oxide Fuel Cell } \\
\text { (194 MW Fuel Cell;106 MW Steam Turbine) }\end{array}$ & Long & 300 & 1 & 800 & 900 & 1000 & 6 & 9 & 5700 & 59.9 & 5 \\
\hline
\end{tabular}

NOTES:

1. NEAR TERM - 1991-1995; LONG TERM - 1996-2007

2. LONO TERM COST AND PERFORMANCE GOALS - COMPLEX OR COMBINED CYCLE

3. TOTAL PLANT COST IS OVERNIGHT COST AND DOES NOT INCLUDE AFUDC, ESCALATION DURING CONSTRUCTION, LAND OR OWNER'S COSTS

4. CAPACTY FACTOR - 80\% FOR ALL CASES

5. REPOWERING STUDIES ARE ONGOING, AND THESE ESTIMATES COULD CHANGE SIGNIFICANTLY 
Table 2

Advanced Gas Intermediate Load Generation Options (1990 Dollars)

\begin{tabular}{|c|c|c|c|c|c|c|c|c|c|c|}
\hline $\begin{array}{c}\text { TABLE } \\
\text { NO. }\end{array}$ & $\begin{array}{l}\text { TECHNOLOGY CASE NAME } \\
\text { (Refer Appendix D Tables "IG1 thru 1G7" } \\
\text { For Detailed Data Sheets) }\end{array}$ & $\begin{array}{l}\text { Commercial } \\
\text { Operation } \\
\text { Time Frame } \\
\text { (Note 1) }\end{array}$ & $\begin{array}{c}\text { Unit } \\
\text { Size } \\
(\mathrm{MWe})\end{array}$ & $\begin{array}{l}\text { Plant } \\
\text { Size } \\
\text { No or } \\
\text { Units }\end{array}$ & $\begin{array}{l}\text { Total Plant } \\
\text { Cost (\$/kW) } \\
\text { (Note 2) }\end{array}$ & $\begin{array}{c}\text { O\&M } \\
\text { Flxed } \\
(\$ / \mathrm{kW}-\mathrm{Yr})\end{array}$ & $\begin{array}{c}\text { O\&M } \\
\text { Variable } \\
(\text { Mills/kWh) }\end{array}$ & $\begin{array}{l}\text { Full Load } \\
\text { Hoat Rate } \\
\text { HHN } \\
\text { (Btu/kWh) }\end{array}$ & $\begin{array}{l}\text { Efficioncy } \\
\text { (HHV-\%) }\end{array}$ & $\begin{array}{l}\text { Quality of } \\
\text { Data (Scale } \\
\text { (1)-Best To } \\
\text { (5)-Worst }\end{array}$ \\
\hline $\begin{array}{l}\text { IG1 } \\
\text { |GI }\end{array}$ & $\begin{array}{l}\text { Combined Cycle Power Plant (Freme 7E) } \\
\text { Combined Cycle Power Plant (Freme 7F) }\end{array}$ & $\begin{array}{l}\text { Near } \\
\text { Long. }\end{array}$ & $\begin{array}{ll}123 \\
222\end{array}$ & $\begin{array}{l}4 \\
2\end{array}$ & 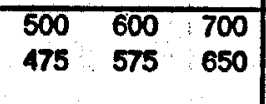 & & $\begin{array}{l}6 \\
6\end{array}$ & $\begin{array}{r}7990 \\
7514\end{array}$ & $\begin{array}{r}42.7 \\
45.4 \\
\vdots\end{array}$ & $\overline{3}$ \\
\hline 163 & Gas Tumbine STIG - LM1600 - 17 MW & Near & 17 & 3 & $800 \quad 900 \quad 1000$ & 10 & 9 & 9320 & 36.6 & 4 \\
\hline 104 & Gas Tubine STla - LM5000 - 50 MW & Near & 51 & 4 & $700 \quad 800 \quad 000$ & 9 & 9 & 8700 & 39.2 & 3 \\
\hline $\begin{array}{l}102 \\
192 a\end{array}$ & $\begin{array}{l}\text { LM6000 Com cyc(two 42 MW GT;one 25MW steam) } \\
\text { Advanced aeroderivative (See Note 3) }\end{array}$ & $\begin{array}{l}\text { Near. } \\
\text { Long }\end{array}$ & $\begin{array}{l}109 \\
160\end{array}$ & 4 & $\begin{array}{|lll|}500 & 600 & 700 \\
600 & 600 & 800\end{array}$ & & & $\begin{array}{r}7770 \\
6100-6700\end{array}$ & $\begin{array}{r}43.9 \\
51.56 \%\end{array}$ & $\begin{array}{l}3 \\
5\end{array}$ \\
\hline $\begin{array}{l}\text { lG5 } \\
\text { lG5 }\end{array}$ & $\begin{array}{l}\text { LM6000 Simple Cycle } \\
\text { Advancod Simple Cycto LM6000 (intercooled) }\end{array}$ & $\begin{array}{l}\text { Near } \\
\text { Long }\end{array}$ & $\begin{array}{l}40 \\
60\end{array}$ & $\begin{array}{l}4 \\
2\end{array}$ & \begin{tabular}{lll|}
450 & 500 & 600 \\
400 & 450 & 550
\end{tabular} & $\begin{array}{l}9 \\
9\end{array}$ & & $\begin{array}{l}9230 \\
8754\end{array}$ & $\begin{array}{l}37.0 \\
39.0\end{array}$ & $\begin{array}{l}3 \\
\end{array}$ \\
\hline $\begin{array}{l}1 \mathrm{G6} \\
1 \mathrm{G6}\end{array}$ & $\begin{array}{l}\text { Phosphoric Acid Fuel Cell } \\
\text { Phosphoric Acid Fuel Cell }\end{array}$ & $\begin{array}{l}\text { Near. } \\
\text { Long }\end{array}$ & $\begin{array}{l}10 \\
25\end{array}$ & $\begin{array}{l}1 \\
4\end{array}$ & \begin{tabular}{lll|}
1400 & 1500 & 1900 \\
1000 & 1000 & 1200
\end{tabular} & & \begin{tabular}{r|}
6.5 \\
5
\end{tabular} & $\begin{array}{l}8300 \\
7000\end{array}$ & $\begin{array}{ll}41.1 \\
48.8\end{array}$ & 3 \\
\hline $\begin{array}{l}\text { 1G7 } \\
107\end{array}$ & $\begin{array}{l}\text { Molten Carbonate Fuel Cell } \\
\text { MCFC (20 MW steam tub bottoming cycle) }\end{array}$ & $\begin{array}{l}\text { Near } \\
\text { Long }\end{array}$ & $\begin{array}{r}2 \\
100 \\
\end{array}$ & $\begin{array}{r}1 \\
1 \\
\end{array}$ & \begin{tabular}{rrr|}
1500 & 2000 & 2500 \\
800 & 1000 & 1200 \\
\end{tabular} & \begin{tabular}{l|}
7 \\
6
\end{tabular} & $\begin{array}{r}10 \\
9 \\
\end{array}$ & $\begin{array}{l}6825 \\
5700 \\
\end{array}$ & $\begin{array}{l}50.0 \\
59.9\end{array}$ & 4 \\
\hline
\end{tabular}

NOTES:

1. NEAA TERM - 1991-1995; LONG TERM - 1996-2007

2. TOTAL PLANT COST IS OVERNIOHT COST AND DOES NOT INCLUDE AFUDC, ESCALATION DURING CONSTRUCTION, LAND OR OWNER'S COSTS

3. LONO TERM COST AND PERFORMANCE GOALS - COMPLEX OR COMBINED CYCLE

4. CAPACTY FACTOR - $35 \%$ FOR ALL CASES 
Table 3

Advanced Gas Distributed Generation Options

(1990 Dollars)

\begin{tabular}{|c|c|c|c|c|c|c|c|c|c|c|c|c|c|}
\hline $\begin{array}{l}\text { TABLE } \\
\text { NO. }\end{array}$ & $\begin{array}{l}\text { TECHNOLOGY CASENAME } \\
\text { (Refer Appendix D Tables "BD1 thru BD4" } \\
\text { and "BDC1 THRU BDC4" for Detalled Data } \\
\text { Sheets) }\end{array}$ & $\begin{array}{l}\text { Commercial } \\
\text { Operation } \\
\text { Time Frame } \\
\text { (Note 1) }\end{array}$ & $\begin{array}{l}\text { Unit } \\
\text { Size } \\
(\mathrm{MW} \text { ) }\end{array}$ & \begin{tabular}{|c|} 
Plant \\
Stze \\
No of \\
Units
\end{tabular} & $\begin{array}{l}\text { Total } \\
\text { Cost } \\
\text { (Note } \\
\text { (Low) }\end{array}$ & $\begin{array}{l}\text { Plant } \\
\text { (\$/KWM } \\
\text { (R) }\end{array}$ & (High) & $\begin{array}{c}\text { O\&M } \\
\text { Fxed } \\
\left(\$ / \mathrm{kW}-\mathrm{Y}_{\mathrm{r}}\right)\end{array}$ & $\begin{array}{c}\text { O\&M } \\
\text { Variable } \\
\text { (MMills/kWh) }\end{array}$ & $\begin{array}{l}\text { Full Load } \\
\text { Heat Pato } \\
\text { HHN } \\
\text { (Btu/kWh) }\end{array}$ & $\begin{array}{l}\text { Effictency } \\
\text { (HHV.\%) }\end{array}$ & $\begin{array}{c}\text { \% Input } \\
\text { Btus } \\
\text { Rocovered } \\
\text { As Useable } \\
\text { Heat }\end{array}$ & $\begin{array}{l}\text { Quality of } \\
\text { Data (Scalo } \\
\text { (1)-Best To } \\
\text { (5)-Worst }\end{array}$ \\
\hline $\begin{array}{l}\text { BD1 } \\
\text { BD1 }\end{array}$ & $\begin{array}{l}\text { Commercial Reciprocating Engine } \\
\text { Commerclal Reclprocating Engine }\end{array}$ & $\begin{array}{l}\text { Near } \\
\text { Long }\end{array}$ & $\begin{array}{l}0.2 \\
0.2\end{array}$ & $\begin{array}{l}1 \\
1\end{array}$ & $\begin{array}{l}610 \\
560\end{array}$ & $\begin{array}{l}710 \\
660\end{array}$ & $\begin{array}{l}1100 \\
1000\end{array}$ & \begin{tabular}{l|l}
8 \\
8
\end{tabular} & $\begin{array}{l}15 \\
13\end{array}$ & $\begin{array}{r}10000 \\
9250\end{array}$ & $\begin{array}{l}34.1 \\
36.9\end{array}$ & & $\begin{array}{l}3 \\
3\end{array}$ \\
\hline $\begin{array}{l}\mathrm{BD2} \\
\mathrm{BD2}\end{array}$ & $\begin{array}{l}\text { Commercial Molten Carbonate Fuel Cell } \\
\text { Commercial Molten Carbonate Fuel Cell }\end{array}$ & $\begin{array}{l}\text { Noar } \\
\text { Long }\end{array}$ & $\begin{array}{l}0.2 \\
0.2\end{array}$ & $\begin{array}{l}1 \\
1 \\
1\end{array}$ & $\begin{array}{l}1500 \\
1100\end{array}$ & $\begin{array}{l}2000 \\
1250\end{array}$ & $\begin{array}{l}2500 \\
1500\end{array}$ & \begin{tabular}{l|}
25 \\
25
\end{tabular} & & $\begin{array}{l}7166 \\
6825\end{array}$ & $\begin{array}{l}47.6 \\
50.0\end{array}$ & & $\begin{array}{l}4 \\
4\end{array}$ \\
\hline $\begin{array}{l}\mathrm{BD3} \\
\mathrm{BD3}\end{array}$ & $\begin{array}{l}\text { Small Industrial Reciprocating Engine } \\
\text { Small Industrial Reciprocating Engine }\end{array}$ & $\begin{array}{l}\text { Near } \\
\text { Long }\end{array}$ & $\begin{array}{l}2 \\
2\end{array}$ & 1 & $\begin{array}{l}850 \\
700\end{array}$ & $\begin{array}{l}950 \\
800\end{array}$ & $\begin{array}{l}1300 \\
1150\end{array}$ & & $\begin{array}{r}10 \\
8\end{array}$ & $\begin{array}{r}10000 \\
9000\end{array}$ & $\begin{array}{l}34.1 \\
37.9\end{array}$ & & 3 \\
\hline $\begin{array}{l}\text { BD4 } \\
\text { BD4 }\end{array}$ & $\begin{array}{l}\text { Small Industrial Molten Carb Fuel Cell } \\
\text { Small Industrial Molten Carb Fuel Cell }\end{array}$ & $\begin{array}{l}\text { Near } \\
\text { Long }\end{array}$ & $\begin{array}{l}2 \\
2\end{array}$ & 1 & $\begin{array}{r}1500 \\
900\end{array}$ & $\begin{array}{l}2000 \\
1000\end{array}$ & $\begin{array}{l}2500 \\
1500\end{array}$ & & $\begin{array}{r}10 \\
9\end{array}$ & $\begin{array}{l}6825 \\
6200\end{array}$ & $\begin{array}{l}50.0 \\
55.1\end{array}$ & & $\begin{array}{l}4 \\
4\end{array}$ \\
\hline $\begin{array}{l}\text { BDC1 } \\
\text { BDC1 }\end{array}$ & $\begin{array}{l}\text { Commerclal Rociprocating Engine (Cogen) } \\
\text { Commercial Reciprocating Engine (Cogen) } \\
\text { (Note 4) }\end{array}$ & $\begin{array}{l}\text { Near } \\
\text { Long }\end{array}$ & $\begin{array}{l}0.2 \\
0.2\end{array}$ & $\begin{array}{l} \\
1\end{array}$ & $\begin{array}{l}775 \\
750\end{array}$ & $\begin{array}{r}1000 \\
900\end{array}$ & $\begin{array}{l}1300 \\
1200\end{array}$ & \begin{tabular}{l|}
10 \\
10
\end{tabular} & $\begin{array}{l}15 \\
13\end{array}$ & $\begin{array}{l}6700 \\
5735\end{array}$ & $\begin{array}{r}51.0 \\
59.5 \\
\text { (Note 6) }\end{array}$ & $\begin{array}{l}33.0 \\
38.0\end{array}$ & $\begin{array}{l}3 \\
3\end{array}$ \\
\hline $\begin{array}{l}\mathrm{BDC2} \\
\mathrm{BDC2}\end{array}$ & $\begin{array}{l}\text { Commerclel Motten Carb Fuel Coll (Cogen) } \\
\text { Commerclal Motten Carb Fuel Cell (Cogen) } \\
\text { (Notes 4,5) }\end{array}$ & $\begin{array}{l}\text { Near } \\
\text { Long }\end{array}$ & $\begin{array}{l}0.2 \\
0.2\end{array}$ & $\begin{array}{l}1 \\
1\end{array}$ & $\begin{array}{l}1650 \\
1210\end{array}$ & $\begin{array}{l}2200 \\
1375\end{array}$ & $\begin{array}{l}2750 \\
1650\end{array}$ & $\begin{array}{l}25 \\
25\end{array}$ & $\begin{array}{l}17 \\
16\end{array}$ & $\begin{array}{l}6163 \\
5801\end{array}$ & $\begin{array}{r}55.4 \\
58.8 \\
(\text { Noto } 6)\end{array}$ & $\begin{array}{l}14.0 \\
15.0\end{array}$ & $\begin{array}{l}4 \\
4\end{array}$ \\
\hline $\begin{array}{l}\mathrm{BDC3} \\
\mathrm{BDC3}\end{array}$ & $\begin{array}{l}\text { Small Industrial Recip Engine (Cogen) } \\
\text { Small Industrial Recip Engine (Cogen) } \\
\text { (Notes 4,5) }\end{array}$ & $\begin{array}{l}\text { Near } \\
\text { Long }\end{array}$ & $\begin{array}{l}2 \\
2\end{array}$ & 1 & $\begin{array}{r}1000 \\
800\end{array}$ & $\begin{array}{r}1100 \\
900\end{array}$ & $\begin{array}{l}1600 \\
1300\end{array}$ & $\begin{array}{l}5 \\
5\end{array}$ & $\begin{array}{l}11 \\
10\end{array}$ & $\begin{array}{l}6500 \\
5580\end{array}$ & $\begin{array}{r}52.5 \\
61.2 \\
\text { (Noto } 6)\end{array}$ & $\begin{array}{l}35.0 \\
38.0\end{array}$ & $\begin{array}{l}3 \\
3\end{array}$ \\
\hline $\begin{array}{l}\text { BDC4 } \\
\text { BDC4 }\end{array}$ & $\begin{array}{l}\text { Small hind Molten Carb Fuel Cell (Cogen) } \\
\text { Small ind Moften Carb Fuel Cell (Cogen) } \\
\text { Notes 4,5) }\end{array}$ & $\begin{array}{l}\text { Near } \\
\text { Long }\end{array}$ & $\begin{array}{l}2 \\
2\end{array}$ & $\begin{array}{l}1 \\
1\end{array}$ & $\begin{array}{l}1650 \\
990\end{array}$ & $\begin{array}{l}2200 \\
1100\end{array}$ & $\begin{array}{l}2750 \\
1650\end{array}$ & & $\begin{array}{r}10 \\
9\end{array}$ & $\begin{array}{l}6091 \\
5334\end{array}$ & $\begin{array}{r}56.0 \\
64.0 \\
(\text { Note } 6)\end{array}$ & $\begin{array}{l}15.0 \\
16.0\end{array}$ & $\begin{array}{l}4 \\
4\end{array}$ \\
\hline $\begin{array}{l}\text { BDC5 } \\
\text { BDC5 }\end{array}$ & $\begin{array}{l}\text { Industrial Gas Turb Cogen (Notes 4,5,6) } \\
\text { Industrial Ges Turbine Cogen (Notes 4,5,6) }\end{array}$ & $\begin{array}{l}\text { Near } \\
\text { Long }\end{array}$ & $\begin{array}{l}50 \\
50\end{array}$ & $\begin{array}{l}1 \\
1\end{array}$ & $\begin{array}{l}624 \\
604 \\
\end{array}$ & $\begin{array}{l}1125 \\
1007\end{array}$ & $\begin{array}{l}1400 \\
1358 \\
\end{array}$ & $\begin{array}{l}15.4 \\
15.4\end{array}$ & $\begin{array}{ll}6.6 \\
5.5\end{array}$ & $\begin{array}{l}67114 \\
5690\end{array}$ & $\begin{array}{l}50.8 \\
60.0\end{array}$ & $\begin{array}{l}41.0 \\
45.0\end{array}$ & 4 \\
\hline
\end{tabular}

NOTES:

1. NEAR TERM - 1991-1995; LONO TERM - 1996-2007

2. CAPACTTY FACTOR - $80 \%$ FOR ALL CASES

3. TOTAL PLANT COST IS OVERNIGHT COST AND DOES NOT INCLUDE AFUDG, ESCALATION DURING CONSTRUCTION LAND OR OWNER'S COSTS

4. HEAT RATES FOR COGENERATION CASES ARE ADJUSTED TO REFLECT A CREDIT FOR RECOVERED THERMAL ENEROY

5. COOENERATION CONFIOURATIONS ARE REPRESENTATIVE ONLY, AND THE ACTUAL HEAT RECOVERABLE AND COSTS WILL

BE HIOHLY DEPENDENT ON THE MACHINE TYPE, CONFIOURATION AND STTE SPECFIC ARPANGEMENTS AND PLANT CAPACTYY FACTOR

6. EFFICIENCY IS THE EFFECTIVE ELECTRICAL EFFICIENCY AFTER A CREDT IS TAKEN FOR THE HEAT RECOVERED.

THE OVERALL PLANT EFFICIENCY FOR BOTH ELECTRICAL AND HEAT IS A HIOHER NUMBER, AND IS NOT SHOWN IN THIS TABLE 
Table 4

\section{Peak Load Generation and Storage Options (1990 Dollars)}

\begin{tabular}{|c|c|c|c|c|c|c|c|c|c|c|}
\hline $\begin{array}{c}\text { TABLE } \\
\text { NO. }\end{array}$ & $\begin{array}{l}\text { TECHNOLOGY CASE NAME } \\
\text { (Refer Appendix D Tables "P1 thru P5 } \\
\text { for Detalled Data Shoets) }\end{array}$ & $\begin{array}{l}\text { Commercial } \\
\text { Operation } \\
\text { Time Frame } \\
\text { (Note 1) }\end{array}$ & $\begin{array}{c}\text { Unit } \\
\text { Size } \\
(M W o)\end{array}$ & $\begin{array}{l}\text { Plant } \\
\text { Size } \\
\text { No of } \\
\text { Units }\end{array}$ & $\begin{array}{l}\text { Total Plant } \\
\text { Cost (\$/kW) } \\
\text { (Note 2) } \\
\text { (Low) (Ret) (High) }\end{array}$ & $\begin{array}{c}\text { O\&M } \\
\text { Fxed } \\
(\$ / \mathrm{kW} \cdot \mathrm{Y})\end{array}$ & $\begin{array}{c}\text { O\&M } \\
\text { Vartable } \\
\text { (Milis/kWh) }\end{array}$ & $\begin{array}{l}\text { Full Load } \\
\text { Heat Rate } \\
\text { HHW } \\
\text { (Btu/kWh) }\end{array}$ & $\begin{array}{l}\text { Efficiency } \\
(H H V \cdot \%)\end{array}$ & $\begin{array}{l}\text { Quality of } \\
\text { Data (Scalo } \\
\text { (1)-Best To } \\
\text { (5)-Worst }\end{array}$ \\
\hline P1 & DOA Combustion Turbine (Gas) & Noar: & 101.5 & 1 & $435 \quad 435,435$ & 6 & 4.45 & 11930 & 28.6 & 2 \\
\hline $\begin{array}{l}\text { P2 } \\
\text { P2 }\end{array}$ & $\begin{array}{l}\text { Current aeroderivative (LM6000-Simple Cycle) } \\
\text { Advanced Simple Cooled LM6000 (intercooled) } \\
\text { (See Note 4) }\end{array}$ & $\begin{array}{l}\text { Near } \\
\text { Long }\end{array}$ & $\begin{array}{l}40 \\
80\end{array}$ & $\begin{array}{l}4 \\
2\end{array}$ & $\begin{array}{rrr}450 & 500 & 600 \\
400 & 450 & 500 \\
\end{array}$ & $\begin{array}{l}8 \\
8\end{array}$ & & $\begin{array}{l}9230 \\
8754\end{array}$ & $\begin{array}{l}37.0 \\
39.0\end{array}$ & $\begin{array}{l}3 \\
5\end{array}$ \\
\hline $\begin{array}{l}\text { P3 } \\
\text { P3 }\end{array}$ & $\begin{array}{l}\text { Lead Acid Battery (Noto 3) } \\
\text { Load Acid Battery }\end{array}$ & $\begin{array}{l}\text { Neer } \\
\text { Long }\end{array}$ & $\begin{array}{l}20 \\
20\end{array}$ & $\begin{array}{l}1 \\
1\end{array}$ & $\begin{array}{lll}650 & 850 & 950 \\
500 & 560 & 670\end{array}$ & $\begin{array}{l}1.4 \\
1.4\end{array}$ & $\begin{array}{l}6.5 \\
6.5\end{array}$ & & & $\begin{array}{l}4 \\
4\end{array}$ \\
\hline P4 & Small Hydroelectric Plant & Near & 10 & 1 & $1000 \quad 2500 \quad 3000$ & 12 & 3.3 & & & 4 \\
\hline P5 & Conventional Pumped Hydro Plant (Note 3) & Near & 333 & 3 & $880 \quad 1150$ & 4 & 5 & & & 4 \\
\hline
\end{tabular}

NOTES:

1. NEAR TERM - 1991-1995; LONQ TERM - 1996-2007

2. TOTAL PLANT COST IS OVERNIOHT COST AND DOES NOT INCLUDE AFUDC, ESCALATION OURINO CONSTRUCTION, LAND OR OWNER'S COSTF

3. STORAGE OPTION WHICH REQUIRES LOW COST CHAROING ELECTRICTTY

4. LONO TERM COST AND PERFORMANCE GOALS

5. CAPACITY FACTOR - $10 \%$ FOR ALL CASES 
Renewable Technologies - Baseload Generation Options (1990 Dollars)

\begin{tabular}{|c|c|c|c|c|c|c|c|c|c|c|}
\hline $\begin{array}{l}\text { TABLE } \\
\text { NO. }\end{array}$ & $\begin{array}{l}\text { TECHNOLOGY CASE NAME } \\
\text { (Refer Appendlx D Tables "Bl1 thru Bl11" } \\
\text { for Detailed Data Sheets) }\end{array}$ & $\begin{array}{l}\text { Cornmercial } \\
\text { Operation } \\
\text { Time Frame } \\
\text { (Note 1) }\end{array}$ & $\begin{array}{l}\text { Unit } \\
\text { Size } \\
(\mathrm{MW})\end{array}$ & $\begin{array}{l}\text { Plant } \\
\text { Size } \\
\text { No of } \\
\text { Units }\end{array}$ & $\begin{array}{l}\text { Total Plant } \\
\text { Cost (\$/kW) } \\
\text { (Note 2) } \\
\text { Low) (Ret) (High) }\end{array}$ & $\begin{array}{c}\text { O\&M } \\
\text { Fbxod } \\
(\$ / \mathrm{KW}-\mathrm{Yr})\end{array}$ & $\begin{array}{c}\text { O\&M } \\
\text { Variable } \\
\text { (Millo/kWh) }\end{array}$ & $\begin{array}{l}\text { Full Loed } \\
\text { Heat Rate } \\
\text { HHV } \\
\text { (Btu/kWh) }\end{array}$ & $\begin{array}{l}\text { Plant } \\
\text { Av Cap } \\
\text { Factor } \\
\text { (\%) }\end{array}$ & $\begin{array}{l}\text { Quality of } \\
\text { Data (Scalo } \\
\text { (1)-Best To } \\
\text { (5)-Worst }\end{array}$ \\
\hline $\begin{array}{l}\text { Bi1 } \\
\text { Bl1 }\end{array}$ & Geothermal - Hot Water (exc1. field costs) & $\begin{array}{l}\text { Near } \\
\text { Long }\end{array}$ & $\begin{array}{l}50 \\
50\end{array}$ & 4 & \begin{tabular}{lll|}
1710 & 1710 & 2050 \\
1500 & 1500 & 1700
\end{tabular} & $\begin{array}{l}58 \\
58\end{array}$ & $\begin{array}{l}4.7 \\
4.7\end{array}$ & $\begin{array}{l}33000 \\
33000\end{array}$ & $\begin{array}{l}80 \\
80\end{array}$ & $\begin{array}{l}3 \\
3\end{array}$ \\
\hline $\begin{array}{l}812 \\
812\end{array}$ & $\begin{array}{l}\text { Geothermal - Hot Water Modular Wellhead } \\
\text { (excluding field costs) }\end{array}$ & $\begin{array}{l}\text { Near } \\
\text { Long }\end{array}$ & $\begin{array}{l}10 \\
20\end{array}$ & $\begin{array}{l}5 \\
5\end{array}$ & $\begin{array}{|lll|}2100 & 2700 & 3300 \\
1000 & 2100 & 2500\end{array}$ & $\begin{array}{l}40 \\
40\end{array}$ & $\begin{array}{l}2 \\
2\end{array}$ & $\begin{array}{l}33000 \\
33000\end{array}$ & $\begin{array}{l}80 \\
80\end{array}$ & $\begin{array}{l}3 \\
3\end{array}$ \\
\hline 813 & Geothermal - Hot Dry Rock (excl.field costs) & Long & 20 & 5 & $1900 \quad 2100 \quad 2500$ & 40 & 2 & 33000 & 80 & $\overline{5}$ \\
\hline B14 & Geothermal Steam (excl. field costa) & Noar & 140 & $T$ & $1500 \quad 1500 \quad 1500$ & 21.7 & 3 & 21544 & 80 & 1 \\
\hline B15 & Large Biomass Plant & Near & 36 & 1 & \begin{tabular}{lll|}
1620 & 1960 & 2900 \\
\end{tabular} & 30 & 12 & 16225 & 80 & 3 \\
\hline$\overline{B 16}$ & Small Biomass Plant (used equipment) & Noar & 5 & 1 & $500 \cdot 750 \cdot 1000$ & 50 & 12 & 17500 & 80 & 3 \\
\hline$\overline{817}$ & Biomass Gasifier STIG (LM5000) & Long & 53 & 1 & $1300 \quad 1500 \quad 2000$ & 30 & 12 & 10500 & 80 & 4 \\
\hline $\begin{array}{l}\text { B18 } \\
\text { B18a } \\
\text { B18b }\end{array}$ & $\begin{array}{l}\text { Municipal Solid Waste to Energy Plant } \\
\text { Municipal Solid Waste (Retrofit-Opt 1A) } \\
\text { Municipal Solid Waste (Retrofit-Opt 1B) }\end{array}$ & $\begin{array}{l}\text { Long } \\
\text { Long } \\
\text { Long }\end{array}$ & $\begin{array}{l}19 \\
38 \\
67\end{array}$ & $\begin{array}{l}1 \\
1 \\
1\end{array}$ & $\begin{array}{|l:ll|}4240 & 5030 & 6540 \\
2530 & 2530 & 2785 \\
1940 & 1940 & 2134 \\
\end{array}$ & $\begin{array}{l}142 \\
225 \\
168\end{array}$ & $\begin{array}{l}8 \\
25 \\
21\end{array}$ & $\begin{array}{l}15000 \\
16300 \\
15500\end{array}$ & $\begin{array}{l}80 \\
80 \\
80\end{array}$ & $\begin{array}{l}3 \\
3 \\
3\end{array}$ \\
\hline 819 & Small Hydroelectric Plant & Near & 5 & 1 & $\begin{array}{|lll|}1370 & 3420 & 4100 \\
\end{array}$ & 23 & 5.5 & & 50 & 3 \\
\hline B110 & Large Hydroelectric Plant & Near & 50 & $\overline{3}$ & $\begin{array}{|lll|}1076 & 4480 & 5377 \\
\end{array}$ & 19.8 & 4.4 & & 40 & 3 \\
\hline $\begin{array}{l}\text { Bi11 } \\
\text { Bl11 }\end{array}$ & $\begin{array}{l}\text { Wind Power Plant } \\
\text { Wind Power Plant }\end{array}$ & $\begin{array}{l}\text { Near } \\
\text { Long }\end{array}$ & $\begin{array}{l}0.2 \\
0.5\end{array}$ & $\begin{array}{l}250 \\
100\end{array}$ & \begin{tabular}{rrr|}
900 & 1100 & 1300 \\
600 & 800 & 1100
\end{tabular} & $\begin{array}{l}23 \\
16\end{array}$ & $\begin{array}{l}.8 \\
2.5 \\
\end{array}$ & & $\begin{array}{l}23 \\
24\end{array}$ & $\begin{array}{l}2 \\
2\end{array}$ \\
\hline
\end{tabular}

NOTES:

1. NEAR TERM - 1991-1995; LONG TERM - 1996-2007

2. TOTAL PLANT COST IS OVERNIOHT COST AND DOES NOT INCLUDE, AFUDC, ESCALATION DURINO CONSTRUCTION, LAND OR OWNER'S COST 
Table 6

Renewable and Storage Technologies - Intermediate Load Generation Options (1990 Dollars)

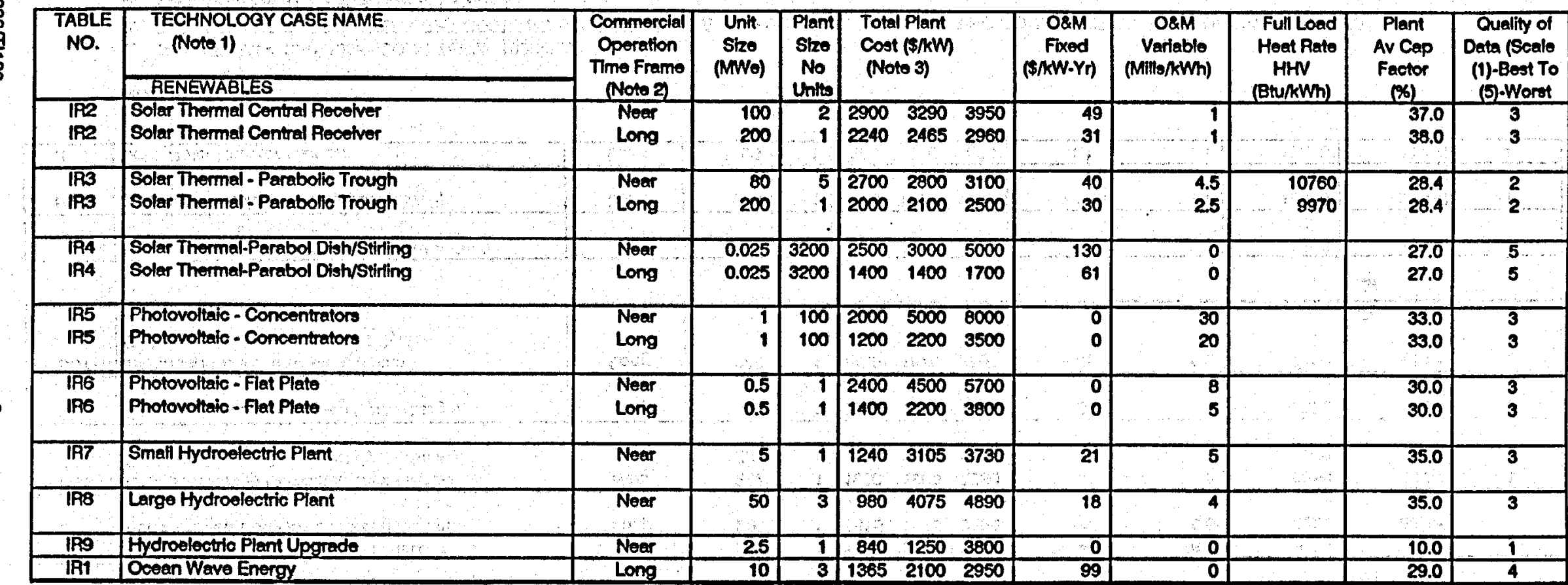

STORAGE TECHNOLOGIES (Note 4 )

\begin{tabular}{|c|c|c|c|c|c|c|c|c|c|c|c|c|c|}
\hline IS1. & Modular Pumped Hydro Plant & Near & 100 & $T$ & & $50 \quad 55$ & 395 & 900 & 1.3 & 1.4 & 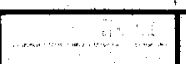 & 20.0 & 4 \\
\hline $\begin{array}{l}152 \\
152\end{array}$ & $\begin{array}{l}\text { Large Compress Air Energy Storage Plant } \\
\text { Large Compress Air Energy Storage Plant }\end{array}$ & $\begin{array}{l}\text { Neer } \\
\text { Long }\end{array}$ & $\begin{array}{l}110 \\
110\end{array}$ & 1 & 39 & $\begin{array}{ll}90 & 56 \\
90 & 56\end{array}$ & 360 & $\begin{array}{l}690 \\
690\end{array}$ & $\begin{array}{l}1.2 \\
1.2\end{array}$ & \begin{tabular}{l|}
2 \\
2
\end{tabular} & $\begin{array}{r}4000 \\
3800\end{array}$ & $\begin{array}{l}20.0 \\
20.0\end{array}$ & $\begin{array}{l}3 \\
3\end{array}$ \\
\hline 153 & Superconducting Magnetic Energy Storage & Long & 1000 & 1 & 79 & 9010 & 301 & 1600 & 5 & 0.2 & & 20.0 & 4 \\
\hline
\end{tabular}

NOTES:

1. NEAR TERM - 1991-1995; LONG TERM - 1996-2007

2. TOTAL PLANT COST IS OVERNIOHT COST AND DOES NOT INCLUDE, AFUDC, ESCALATION DURINO CONSTRUCTION, LAND OR OWNER'S COST

3. STORAGE TECHNOLOGIES WILL AEQUIRE LOW COST ELECTRICITY CHAROING 
Table 7

Advanced Coal and Nuclear - Baseload Central Station Options (1990 Dollars)

\begin{tabular}{|c|c|c|c|c|c|c|c|c|c|c|}
\hline $\begin{array}{l}\text { TABLE } \\
\text { NO. }\end{array}$ & $\begin{array}{l}\text { TECHNOLOQY CASE NAME } \\
\text { (NOIe 1) } \\
\text { COAL } \\
\end{array}$ & $\begin{array}{c}\text { Commercial } \\
\text { Operetion } \\
\text { Time Frame } \\
\text { (Note 2) }\end{array}$ & $\begin{array}{c}\text { Unit } \\
\text { Size } \\
\text { (MWe) }\end{array}$ & \begin{tabular}{|l|} 
Plant \\
Sizo \\
No of \\
Units
\end{tabular} & $\begin{array}{l}\begin{array}{l}\text { Total Plant } \\
\text { Cost (\$/kW) } \\
\text { (Note 3) }\end{array} \\
\text { (Low) (Ref) (High) }\end{array}$ & $\begin{array}{c}\text { O\&M } \\
\text { Fixed } \\
(\$ / k W-Y \text { ) }\end{array}$ & $\begin{array}{c}\text { O\&M } \\
\text { Variable } \\
\text { (Mills/kWh) }\end{array}$ & $\begin{array}{l}\text { Full Load } \\
\text { Heat Rate } \\
\text { HHV } \\
\text { (Btu/kWh) }\end{array}$ & $\begin{array}{c}\text { Efficiency } \\
(H H N-\%)\end{array}$ & $\begin{array}{l}\text { Quality of } \\
\text { Data (Scale } \\
\text { (1)-Best To } \\
\text { (5)-Worst }\end{array}$ \\
\hline BC1 & Curent Pulverized Coal (Smali) & Near & 275 & 1 & $1820 \quad 2135 \quad 2350$ & 43 & 5.5 & 9750 & $35.0 \%$ & 3 \\
\hline $\mathrm{BC2}$ & Advanced Pulverized Coal (Small) & Noar & 204 & 1 & $1920 \quad 2255 \quad 2480$ & 45 & $\overline{5.5}$ & 8821 & $38.7 \%$ & 3 \\
\hline $\mathrm{BC3}$ & Advanced Pulverized Coel (Large) & Long & 606 & 1 & \begin{tabular}{|lll}
1420 & 1670 & 1840
\end{tabular} & 26.5 & 4.4 & 8446 & $40.4 \%$ & $\overline{3}$ \\
\hline $\begin{array}{l}\text { BCA } \\
B C 4\end{array}$ & $\begin{array}{l}\text { Atm Fluidized Bed Combustion (Medium) } \\
\text { Atm Fluidized Bed Combustion (Medium) }\end{array}$ & $\begin{array}{l}\text { Near } \\
\text { Long }\end{array}$ & $\begin{array}{l}200 \\
200\end{array}$ & $\begin{array}{l}1 \\
1\end{array}$ & $\begin{array}{|lll|}1800 & 2195 & 2525 \\
1615 & 1995 & 2295 \\
\end{array}$ & $\begin{array}{l}45 \\
45\end{array}$ & $\begin{array}{l}6.6 \\
6.6\end{array}$ & $\begin{array}{l}9765 \\
8825\end{array}$ & $35.0 \%$ & 3 \\
\hline $\begin{array}{l}\text { BC5 } \\
\text { BC5 }\end{array}$ & $\begin{array}{l}\text { Coal Gasification Combined Cycle (Small) } \\
\text { Coal Gasification Combined Cycle (Small) }\end{array}$ & $\begin{array}{l}\text { Near } \\
\text { Long }\end{array}$ & $\begin{array}{l}227 \\
203\end{array}$ & $\begin{array}{l}1 \\
1\end{array}$ & 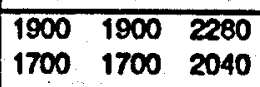 & $\begin{array}{l}45 \\
40\end{array}$ & $\begin{array}{l}5 \\
5\end{array}$ & $\begin{array}{l}9350 \\
9000\end{array}$ & $\begin{array}{l}36.5 \% \\
37.9 \%\end{array}$ & $\overline{3}$ \\
\hline BC6 & Coal Gasfication Combined Cycle (Large) & Long & 410 & 1 & $\begin{array}{|lll|}1600 & 1600 & 1920\end{array}$ & 38 & $\overline{4}$ & 8850 & $38.6 \%$ & $\overline{3}$ \\
\hline BC7 & $\begin{array}{l}\text { Integrated Coal Gasification/Molten } \\
\text { Carbonate Fuel Cell Combined Cycle }\end{array}$ & Long & 200 & 1 & \begin{tabular}{|ll|l}
1780 & 1870 & 2500 \\
\end{tabular} & 45 & 5.5 & 7100 & $48.1 \%$ & 4 \\
\hline
\end{tabular}

NUCLEAR

\begin{tabular}{|c|c|c|c|c|c|c|c|c|c|c|}
\hline BN1 & High Temperature Ges-Cooled (Nth of a Kind) & Long & 175 & $\overline{4}$ & $\begin{array}{lll}1586 & 1586 & 2062\end{array}$ & 55 & 0.2 & 8860 & $38.5 \%$ & 4 \\
\hline BN2 & Advanced Passive LWR (AP600, ABWR) & Long & 600 & 1 & $\begin{array}{lll}475 & 1475 & 1770\end{array}$ & 72.7 & 1.1 & 10220 & $33.4 \%$ & 4 \\
\hline BN3 & Light Water Reactor (Current) & Long & 1200 & $T$ & $1300 \quad 1300 \quad 1600$ & 61.1 & 1.1 & 10220 & $33.4 \%$ & 4 \\
\hline
\end{tabular}

NOTES:

1. NEAR TERM - 1991-1995; LONO TERM - 1996-2007

2. TOTAL PLANT COST IS OVERNIGHT COST AND DOES NOT INCLUDE, AFUDC, ESCALATION DURINO CONSTRUCTION, LAND OR OWNER'S COST

2. TOTAL PLANT COST IS OVERNIGHT COST AN 


\section{RENEWABLES}




\section{GEOTHERMAL ENERGY}

Geothermal energy is the heat of the earth. Resources are typically classified as hydrothermal (steam or hot water), hot dry rock, geopressured, and magma. All four resources exist in Northern California. Only hydrothermal resources are currently used commercially for power production. Dry steam resources are very rare. Geothermal energy resources offer the potential for increasing PG\&E's resource diversity in an environmentally acceptable manner. Representative costs of recent commercial geothermal projects are summarized in Table 13. Major insights are:

- PG\&E and several other utilities developed over 2,000 MW of dry steam geothermal plants at The Geysers, the largest geothermal facility in the world. Dry steam plants use mature, relatively conventional steam turbines. Plant costs for the most recent units were close to $\$ 2,000 / \mathrm{kW}$, with unit sizes over $120 \mathrm{MW}$ including $30 \%$ for steam field related costs. The plants are operated in a baseload duty cycle, with capacity factors of over $80 \%$. Conversion efficiencies exceed 22,000 Btu/kWh because of relatively low steam temperatures $\left(350^{\circ} \mathrm{F}\right)$.

- Flash and binary plants utilize lower temperature, liquid-dominated geothermal resources. Flash technology employs similar turbines, but utilizes steam produced by reducing pressure on hot water/brine fluids (flashing). Binary plants use heat in geothermal fluids to vaporize a separate working fhid, via heat exchangers, to tum a turbine in a closed cycle (fluids never come into contact with the atmosphere). Recent flash plants have cost about $\$ 2,500$ to $\$ 3,000 / \mathrm{kW}$, including well costs. Binary plant costs are somewhat higher for individual projects. Binary units do not have large economies of scale, and costs reflect relatively small unit sizes (2-10 MW). Binary plants may be fabricated in a shop and erected in the field to keep costs low. Both have been commercially demonstrated and deployed almost exclusively by independent power producers, mostly in Southern Califomia.

- The Department of Energy, (DOE) Geothermal R\&D Program is primarily focused on hydrothermal technology (exploration, drilling, and binary power conversion), hot dry rock, and geopressured geothermal. Performance and cost improvements in binary cycles in the range of $20-30 \%$ are targeted. DOE is planning a 1992 initiative to demonstrate higher efficiency hydrothermal technology. However, a next generation technology is not clearly defined. Hot dry rock technology is undergoing a 30-MW thermal technical demonstration at Fenton Hill, New Mexico. Research into magma and geopressured geothermal is largely exploratory. The Electric Power Research Institute (EPRI) no longer sponsors an advanced geothermal R\&D program.

- Approximately 20,000 MW of hydrothermal resource potential are thought to exist in the Pacific Northwest, in Southern Califomia, and east of the Sierra Nevada. The Pacific Northwest has not had any power plant development to date, although some resource assessment and exploratory drilling have occurred. BPA is currently planning three separate 10-MW projects to confirm the resource potential of several large fields. Recent exploration at Medicine Lake. California, near the Oregon border indicates a potentially significant resource is relatively near transmission lines running to Northem California. One of the BPA demonstration projects could be sited there. Resource development east of the Sierra Nevada will be limited by transmission access to major power markets, particularly Northern Califomia. 
Table 13

Capltal Costs for Recently Constructed Geothermal Plants

\begin{tabular}{|c|c|c|c|c|c|}
\hline \multirow[b]{2}{*}{ Plant } & \multirow{2}{*}{$\begin{array}{c}\text { Date of } \\
\text { Commercial } \\
\text { Operation } \\
\end{array}$} & \multirow[b]{2}{*}{$\begin{array}{c}\text { Gross } \\
\text { Capactly (MW) }\end{array}$} & \multicolumn{3}{|c|}{ Costs Not Converted to Any Common Year Basis } \\
\hline & & & $\begin{array}{l}\text { Plant Capltal } \\
\text { Cost (\$/kW net) }\end{array}$ & $\begin{array}{l}\text { Fiold Capltal } \\
\text { Cost }(\$ / k W)\end{array}$ & $\begin{array}{l}\text { Total Capltal } \\
\text { Cost (\$k/W) }\end{array}$ \\
\hline \multicolumn{6}{|l|}{ Steam Plant } \\
\hline $\begin{array}{l}\text { PG\&E Unit } 16 \\
\text { PG\&E Unit } 20 \\
\text { PG\&E Unit 21b } \\
\text { Bear Canyon } \\
\text { CCPA }\end{array}$ & $\begin{array}{r}1985 \\
1985 \\
1989 \\
1988\end{array}$ & $\begin{array}{r}120 \\
120 \\
151 \\
22 \\
132\end{array}$ & $\begin{array}{r}1,088 \\
911 \\
1,500 \\
1,550 \\
1,500\end{array}$ & $\begin{array}{l}466^{a} \\
390^{a} \\
643^{a} \\
643^{a} \\
643^{a}\end{array}$ & $\begin{array}{l}1,554^{a} \\
1,301^{a} \\
2,143^{a} \\
2,143^{a} \\
2,143^{a}\end{array}$ \\
\hline \multicolumn{6}{|l|}{ Flash Plant } \\
\hline $\begin{array}{l}\text { Coso \#1c } \\
\text { Coso Project }(230 \mathrm{MW}) \\
\text { Blundell \#1 } \\
\text { Leathers \#1 }\end{array}$ & $\begin{array}{l}1987 \\
1989 \\
1984 \\
1989\end{array}$ & $\begin{array}{r}30 \\
230 \\
23 \\
38\end{array}$ & $\begin{array}{l}1,715 \\
1,820^{\mathrm{g}} \\
1,800 \\
2,100^{\mathrm{a}}\end{array}$ & $\begin{array}{l}715 \text { (net) } \\
780^{\mathrm{a}} \\
770^{\mathrm{a}} \\
900^{\mathrm{a}}\end{array}$ & $\begin{array}{l}2,430 \text { (net) } \\
2,600 \text { (net) } \\
2,570^{8} \\
3,000 \text { (net) }\end{array}$ \\
\hline \multicolumn{6}{|l|}{ Binary } \\
\hline $\begin{array}{l}\text { ORMESA I } \\
\text { Wendel-Amendee }\end{array}$ & $\begin{array}{l}1987 \\
1988\end{array}$ & $\begin{array}{r}30 \\
3\end{array}$ & $\begin{array}{l}2,100^{\mathrm{a}} \\
1,500\end{array}$ & $\begin{array}{l}900^{a} \\
640^{\mathrm{a}}\end{array}$ & $\begin{array}{l}3,000 \text { (gross) } \\
2,140^{8}\end{array}$ \\
\hline
\end{tabular}

aBased on allocating $70 \%$ to plant and $30 \%$ to field.

bPlant never constructed; projected cost used.

cBased on California Energy Company literature. 
- The potential for further hydrothermal development in PG\&E's service area is relatively small (300-MW range), and consists of primarily hot water resources in dispersed and unconfirmed fields. Only one 2-MW binary cycle plant has been developed outside of The Geysers. The Clear Lake area represents the largest identified potential resource in Northern California, with a potential of up to $900 \mathrm{MW}$ of hot dry rock and some hot water resources. The city of Clearlake and the California Energy Commission are in the process of assessing this resource, which might be the next site for commercializing hot dry rock technology if the DOE Fenton Hill Hot Dry Rock Long-Term Flow Test Project in New Mexico is successful.

- Geothermal energy is relatively environmentally benign; however, many of the best geothermal sites are in environmentally sensitive areas and can be difficult or impossible to develop. (e.g., Lassen National Park).

- Power output at The Geysers had dropped from a peak of 1,350 MW (PG\&E-owned units) to around $800 \mathrm{MW}$ by mid-1991 as the result of a decline of the steam field. Output is forecasted to decline to $400 \mathrm{MW}$ by the mid-1990s and to under $200 \mathrm{MW}$ in the post-2000 time frame. Non-PG\&E-owned Geysers units are experiencing similardifficulties. Approaches being investigated to halt the decline include innovative plant operation regimes, new reservoir management techniques, improved reservoir modeling, additional water supply for reinjection, and dry cooling and binary cycle retrofit concepts.

- PG\&E's Advanced Energy Systems R\&D Program has previously participated in the DOE Heber binary cycle geothermal project and in an evaluation of EPRI's advanced modular wellhead binary system. PG\&E's current technology assessment efiorts have focused on characterizing the regional geothermal resources and identifying technology R\&D needs. Modular, higher efficiency binary cycles could help to confirm and lower the development risks for the remaining dispersed and small hot water reservoirs in Northern California. HDR development at Clear Lake might represent a project opportunity if the Fenton Hill tests are successful. Advanced geothermal and high efficiency natural gas hybrid configurations might offer attractive market features for hydrothermal resources outside the service area, possibly a joint project to confirm the resource at Medicine Lake.

- Advanced geothermal has not been targeted as a major new generation opportunity technology for demonstration by PG\&E's R\&D Program because of the relatively mature nature of the hydrothermal technology, the limited potential for further hydrothermal resource development in the service area, and the embryonic state of development of hot dry rock technology.

\section{TECHNOLOGY DESCRIPTION}

Several power conversion systems, all utilizing the Rankine cycle, are used to convert heat from geothermal resources to electric energy. Systems currently in operation are described below.

\section{Direct Steam}

The direct steam system is used with "dry steam" geothermal resources that almost exclusively contain water vapor (steam) with little orno liquid water. After removing rocks and/orother debris, steam trom the geothermal reservoir is piped directly to a steam turtine. The expanding steam spins the turbine rotor and electric generator. Exhaust steam from the furbine is condensed. The resulting liquid (condensate) is used as cooling water and/or reinjected into the reservoir. 


\section{Flash Steam (Single and Dual)}

Flash systems utilize hot water geothermal resources. Hot water or hot water/steam mixtures produced from the reservoir is piped to a flash tank, where the pressure is reduced to produce additional steam. In single flash systems, steam is piped to a turbine for electric generation as for steam turbines. After flashing, the (still) hot brine is reinjected into the reservoir.

For a dual flash system, exhausted brine (from the first flash) is piped to a second flash tank, where the pressure is further reduced to generate more steam, but at a lower pressure than at the first stage. This lower pressure steam is piped either to a lower stage of the turbine or to a separate turbine to generate additional electric power.

Dual flash systems generally have higher thermodynamic efficiencies and higher capital cost. Single flash systems are generally utilized if the geothermal tuid temperature is greater than about $500^{\circ} \mathrm{F}$. Dual flash systems generally are used if the geothermal fluid temperature is $350^{\circ} \mathrm{F}$ to $500^{\circ} \mathrm{F}$. Use of single flash versus dual flash systems may also depend on plant size and equipment availability.

Binary

Whereas the direct steam and flash steam systems utilize the actual geothermal fluid to spin a turbine generator, binary systems utilize a separate working fluid (thus the name binary). Geothermal fluid heats and boils the binary system's working fluid in a heat exchanger. The binary working fluid vapor spins the generator as it expands through the turbine. The working fluid vapor exhausted from the turbine is condensed and pumped up to turbine inlet pressure, to repeat the cycle. Binary working fluids have generally been Freons or hydrocarbons (isobutane and/or isopentane). The optimum working fluid depends on geothermal fluid temperature.

Any hot fluid could be used to heat binary working fluids; however, only hot water geothermal resources have been used to date. Binary plants are more efficient than steam plants at moderate temperatures. They have been installed for geothermal fluid temperatures ranging from $220^{\circ} \mathrm{F}$ to $360^{\circ} \mathrm{F}$.

Binary plants can be designed as modules ranging in size from $300 \mathrm{~kW}$, an example of which is the EPRI modular wellhead binary plant design that utilizes a $300^{\circ} \mathrm{F}$ to $450^{\circ} \mathrm{F}$ range of geothermal fluid temperatures with a single basic turbine design. Power generation ranges from 2.7 MWe to 5.3 MWe net per module depending on geothermal fluid temperature and heat rejection (wet or dry). ORMAT manufactures and installs modular, fully commercial binary plants. It has approximately $100 \mathrm{MW}$ on line. 


\section{PG\&E APPLICATIONS}

Potential geothermal energy applications primarily include baseload power generation. Other plant operation regimes (e.g., seasonal dispatch) may be practical. Hybrid natural gas/geothermal plant operations may provide cost-effective energy, as may plant designs incorporating direct use of lower temperature waste heat from geothermal power production. Some potential for direct use of geothemal heat or waste heat from geothermal power generation exists within PG\&E's service territory. Heat could be used for such applications as central heating systems, hothouse heating, aquaculture, and crop drying operations; however these are typically seasonal thermal loads. There is potential for use of geothermal energy for thermal enhanced oil recovery in Kem County.

\section{DEVELOPMENT AND COMMERCIALIZATION STATUS}

PG\&E and several other utilities developed over 2,000 MW of dry steam geothermal plants at The Geysers, the largest geothermal facility in the world. Dry steam technology is mature and uses relatively conventional steam turbines. If the cost of the production wells are included, plants costs for the most recent units were close to $\$ 2,000 \mathrm{~kW}$, with unit sizes over $120 \mathrm{MW}$. The power plant and the steam fields are operated as separate businesses. The steam field represents roughly $30 \%$ of the capital cost. The plants are operated in a baseload duty cycle, with capacity factors of over $80 \%$. Conversion efficiencles are relatively low (over $22,000 \mathrm{Btu} / \mathrm{kWh}$ ) because of relatively low steam temperatures (350 $\mathrm{F}$ ).

Flash and binary plants utilize lower temperature, liquid-dominated geothermal resources. Flash technology employs similar turbines, but utilizes steam produced by reducing pressure on hot water/brine fluids (flashing). Binary plants use heat in geothermal fluids to vaporize a separate working fluid, via heat exchangers, to turn a turbine in a closed cycle (fluids never come into contact with the atmosphere). Recent flash plants have cost about $\$ 2,500$ to $\$ 3,000 \mathrm{~kW}$ if well costs are included. Binary plant costs are somewhat higher for individual projects. Both have been commercially demonstrated and deployed almost exclusively by independent power producers, mostly in Nevada, Utah, and Southern California.

\section{GEOTHERMAL RESOURCES}

Types of Geothermal Resources

Geothermal fluids can be produced from natural or man-made reservoirs. Most reservoirs naturally contain hot water or hot brine (comprised of water, salt, and other substances) in permeable rock. Naturally occurring hot water is extracted from the reservoir for electric generation. Normally, several wells must be drilled into the reservoir to extract geothermal fluids. Tables 14 to 17 provide a summary of hydrothermal and hot dry rock resources in or near PG\&E's service area. 
Table 14

Hydrothermal Resources Within PG\&E's Service Area

\begin{tabular}{|c|c|c|c|c|c|}
\hline KGRA or Resource & $\begin{array}{l}\text { USGS } 790 \\
\text { Mean Res. } \\
\text { Temp. ( }{ }^{\circ} \text { ) }\end{array}$ & $\begin{array}{c}\text { Estimates } \\
\text { Energy } \\
\text { (MWe/30 Yr) }\end{array}$ & $\begin{array}{c}\text { Measd. } \\
\text { Res. } \\
\text { Temp. ('F) }\end{array}$ & $\begin{array}{c}\text { Current } \\
\text { Capacity } \\
\text { Gross/Net } \\
\text { (MWe) }\end{array}$ & $\begin{array}{l}\text { Plant } \\
\text { Type }\end{array}$ \\
\hline \multicolumn{6}{|l|}{ Dry Steam Reservolrs } \\
\hline $\begin{array}{l}\text { The Geysers } \\
\text { Lassen }\end{array}$ & $\begin{array}{l}459 \\
459\end{array}$ & $\begin{array}{r}1,610 \\
676\end{array}$ & 470-590 & 2,115/1,970 & ${ }^{\mathrm{DS}}$ \\
\hline Total & & 2,286 & & $2,115 / 1,970$ & \\
\hline \multicolumn{6}{|l|}{ Hot Water Reservoirs } \\
\hline Big Bend Hot Springs & 240 & 16 & - & - & - \\
\hline Calistoga Hot Springs & 290 & 40 & - & - & - \\
\hline Chalk Mountain & 235 & 15 & - & - & - \\
\hline Clear Lake/Sulfur Bank Mine & 380 & 975 & - & - & - \\
\hline Kelly Hot Springs & 245 & 16 & - & - & - \\
\hline Morgan/Growler Springs & 425 & 116 & - & - & - \\
\hline Scovern Hot Springs & 225 & 13 & - & - & - \\
\hline Sespe Hot Springs & 270 & 17 & - & - & - \\
\hline Sierra Valley & 260 & 50 & - & - & - \\
\hline Wendell-Amadee & 260 & 54 & $220-230$ & $3.6 / 2.7$ & $B^{d}$ \\
\hline West Valley & 290 & 20 & - & - & - \\
\hline Wilbur Springs & 290 & 73 & - & - & - \\
\hline Total & & 1,405 & & $3.6 / 2.7$ & \\
\hline
\end{tabular}

Unless otherwise noted, temperatures and reservoir potential estimates are from PG\&E, Indigenous Energy Resource Assessment, 1988; J. D. Geyer, L. M. Kelleman, and R. G. Bloomquist, Assessment of Geothermal Resources for Electric Generation in the Pacific Northwest, Northwest Power Planning Council, 1989.

${ }^{b} B=$ Binary. DS = Direct Steam.

Installed capacity - due to steam production decline, actual operating capacity is less (G. R. Priest and G. L. Black, "Geothermal Exploration in Oregon, 1988," Oregon Geology, Vol. 51. No. 5, 1989).

'A 30-MWe net woodwaste/geothermal hybrid plant is operating at Susanville, California. Geothemal fluid is used for feedwater heating. About 1.5 MWe of the total plant output is credited to geothermal energy. 
Table 15

Hydrothermal Resources in the Salton Trough, California

\begin{tabular}{|c|c|c|c|c|c|}
\hline KGRA or Resource & $\begin{array}{c}\text { USGS } 790 \\
\text { Mean Res. } \\
\left.\text { Temperature ( }{ }^{\circ} \mathrm{F}\right)\end{array}$ & $\begin{array}{c}\text { Estimated } \\
\text { Energy } \\
\text { (MWe/30 Yr) }\end{array}$ & $\begin{array}{c}\text { Measured } \\
\text { Res. } \\
\text { Temperature ( }{ }^{\circ} \mathrm{F} \text { ) }\end{array}$ & $\begin{array}{c}\text { Current } \\
\text { Capacity } \\
\text { (Gross/Net } \\
\text { MWe) }\end{array}$ & $\begin{array}{l}\text { Plant } \\
\text { Type }^{6}\end{array}$ \\
\hline Border & 320 & 31 & - & 一 & - \\
\hline Brawley & 487 & 640 & $400-500$ & $-c$ & IF \\
\hline Dunes & 270 & 46 & - & - & - \\
\hline $\begin{array}{l}\text { East Mesa } \\
\text { Fast Mesa }\end{array}$ & 360 & 360 & $293-350$ & $\begin{array}{l}85 / 65 \\
44 / 40\end{array}$ & $\begin{array}{l}B \\
2 F\end{array}$ \\
\hline Glamis & 270 & 43 & - & - & - \\
\hline $\begin{array}{l}\text { Heber } \\
\text { Heber }\end{array}$ & 347 & 650 & 360 & $-d$ & $\begin{array}{l}B \\
2 F\end{array}$ \\
\hline $\begin{array}{l}\text { Salton Sea } \\
\text { Salton Sea }\end{array}$ & 613 & 3,400 & $520-575$ & $\begin{array}{r}10 / 10 \\
225 / 200\end{array}$ & $\begin{array}{l}\text { IF } \\
\text { 2F }\end{array}$ \\
\hline Westmoreland & 423 & 1,710 & - & - & - \\
\hline Total & & 6,880 & & $416 / 362^{\circ}$ & \\
\hline
\end{tabular}

The USGS did not estimate electric energy for resources less than $150^{\circ} \mathrm{C}\left(302^{\circ} \mathrm{F}\right)$. These estimates are based on USGS wellhead thermal energy estimates using conversion factors from J. D. Geyer, L. M. Kellerman, and R. G. Bloomquist, Assessment of Geothermal Resources for Elactric Generation in the Pacific Northwest, Northwest Power Planning Council, 1989.

$b_{B}=$ Binary, $1 F=$ Single Flash, $2 F=$ Dual Flash

A 10-MWe gross/9-MWe net if demonstration plant was operated at Brawley from 1980 to 1986.

-A 61-MWe gross/44-MWe net demonstration binary plant was operated at Heber from 1985 to 1887.

-OESI has announced a 37-MWe net binary plant to be installed at Heber. Magma has announced a total 34-MWe expansion from Magma's four 2F plants at Salton Sea. 
Table 16

Hydrothermal Resources in the Basin and Range, Oregon Plateaus,
Snake River Plain, and Northern Rockles Provinces

\begin{tabular}{|c|c|c|c|c|c|}
\hline KGRA or Resource & $\begin{array}{c}\text { USGS } 790 \\
\text { Mean Res. } \\
\left.\text { Temperature ( }{ }^{\circ} \mathrm{F}\right)\end{array}$ & $\begin{array}{c}\text { Estimated } \\
\text { Energy } \\
\text { (MWe/30 Yr) }\end{array}$ & $\begin{array}{c}\text { Measured } \\
\text { Res. } \\
\left.\text { Temperature ( }{ }^{\circ} \mathrm{F}\right)\end{array}$ & $\begin{array}{c}\text { Current } \\
\text { Capacity } \\
\text { (Gross/Net } \\
\text { MWe) }\end{array}$ & $\begin{array}{l}\text { Plant } \\
\text { Type }^{\text {b }}\end{array}$ \\
\hline Alvord Desert, OR & $358-401$ & 300 & 305 & - & - \\
\hline Baltazor, NV & 316 & 46 & - & - & - \\
\hline Beowawe, NV & 444 & 127 & 420 & $17 / 15$ & $2 F$ \\
\hline Big Creek Springs, ID & 324 & 26 & - & - & - \\
\hline Brady, Hot Springs, NV & 331 & 157 & 309 & 一 & - \\
\hline Colado, NV & 207 & 13 & 300 & - & - \\
\hline Coso Hot Springs, CA & 428 & 650 & $400-700$ & $264 / 240$ & $2 F$ \\
\hline Cove Fort, UT & 333 & 330 & $260 / 340$ & $14 / 10$ & H\&DS \\
\hline Crane/Cove Croek, ID & 340 & 360 & - & - & - \\
\hline Crump Hot Springs, OR & 333 & 61 & - & - & - \\
\hline Darrough Hot Springs, NV & 270 & 74 & - & - & - \\
\hline Desert Peak, NV & 430 & 750 & 400 & $10 / 9$ & $2 F$ \\
\hline Dixie Valley, NV & c & c & $415-482$ & $60 / 55$ & 2F \\
\hline Fallon Navy Base, NV & c & c & - & - & - \\
\hline Fernely area, NV & 360 & 33 & - & - & - \\
\hline Fish Lake, NV & c & c & 315 & - & - \\
\hline Gerlach/Empire, NV & 352 & 32 & $?$ & $5 / 3$ & B \\
\hline Humboldt House/Rye Patch, NV & 423 & 47 & $360-379$ & - & - \\
\hline Kyle Hot Springs, NV & 318 & 97 & - & - & - \\
\hline Lakeview, OR & 302 & 105 & 217 & & B \\
\hline Leach Hot Springs, NV & 324 & 77 & - & - & - \\
\hline Lee Hot Springs, NV & 331 & 28 & - & - & - \\
\hline Long ValleyMammoth, CA & 441 & 2,100 & $330-352$ & $40 / 31$ & $\mathbf{B}$ \\
\hline McCoy, NV & c & c & 216 & - & $=$ \\
\hline Neal Hot Springs, Or & 370 & 36 & - & - & - \\
\hline Pinto Hot Springs, NV & 343 & 90 & - & - & - \\
\hline Ratt River, ID & 300 & 138 & $279-300$ & & B \\
\hline Randsburg area, CA & 342 & 84 & - & - & - \\
\hline Roosevelt Hot Springs, UT & 509 & 970 & $482-520$ & $24 / 22$ & 1F \\
\hline Ruby Valley, NV & 352 & 74 & - & - & - \\
\hline Salt Wells, NV & c & c & $260-350$ & - & - \\
\hline San Emidio Desert, NV & 341 & 28 & 298 & - & - \\
\hline Soda Lake, NV & 319 & 146 & $342-370$ & 19/16 & B \\
\hline Steamboat Springs, NV & 392 & 350 & $340-442$ & $9 / 7$ & B \\
\hline Steamboat Springs, NV & & & & $.13 / 12$ & $\mathbf{1 F}$ \\
\hline Stillwater, NV & 318 & 450 & $310-340$ & $17 / 13$ & B \\
\hline Surprise Valley, CA & 302 & 1,490 & 338 & - & - \\
\hline Thermo, UT & 288 & 12 & - & - & - \\
\hline Trout Creek, OR & 309 & 24 & - & - & - \\
\hline Tuscarora, NV & 329 & 27 & 297 & - & - \\
\hline Vale, OR & 315 & 870 & - & - & - \\
\hline Wabuska, NV & 268 & 94 & 223 & 1.711 .2 & B \\
\hline Wendel-Amadee & 259 & 54 & $220-230$ & $3.6 / 2.7$ & B \\
\hline Total & & $>10,350$ & & $497 / 438$ & \\
\hline
\end{tabular}

The USGS did not estimate electric energy for resources less than $150^{\circ} \mathrm{C}\left(302^{\circ} \mathrm{F}\right)$. Estimates are based on USGS wellhead thermal energy estimates using conversion factors from J. D. Geyer, L. M. Kellerman, and R. G. Bloomquist, Assessment of Geothermal Resources for Electric Generation in the Pacific Northwest, Northwest Power Planning Council, 1989.

$\mathrm{BB}=$ Binary, 1F = Single Flash, $2 F=$ Dual Flash, $H=$ Hybrid.

Not included in USGS Report 790. 
Table 17

Hydrothermal Resources in the Cascades

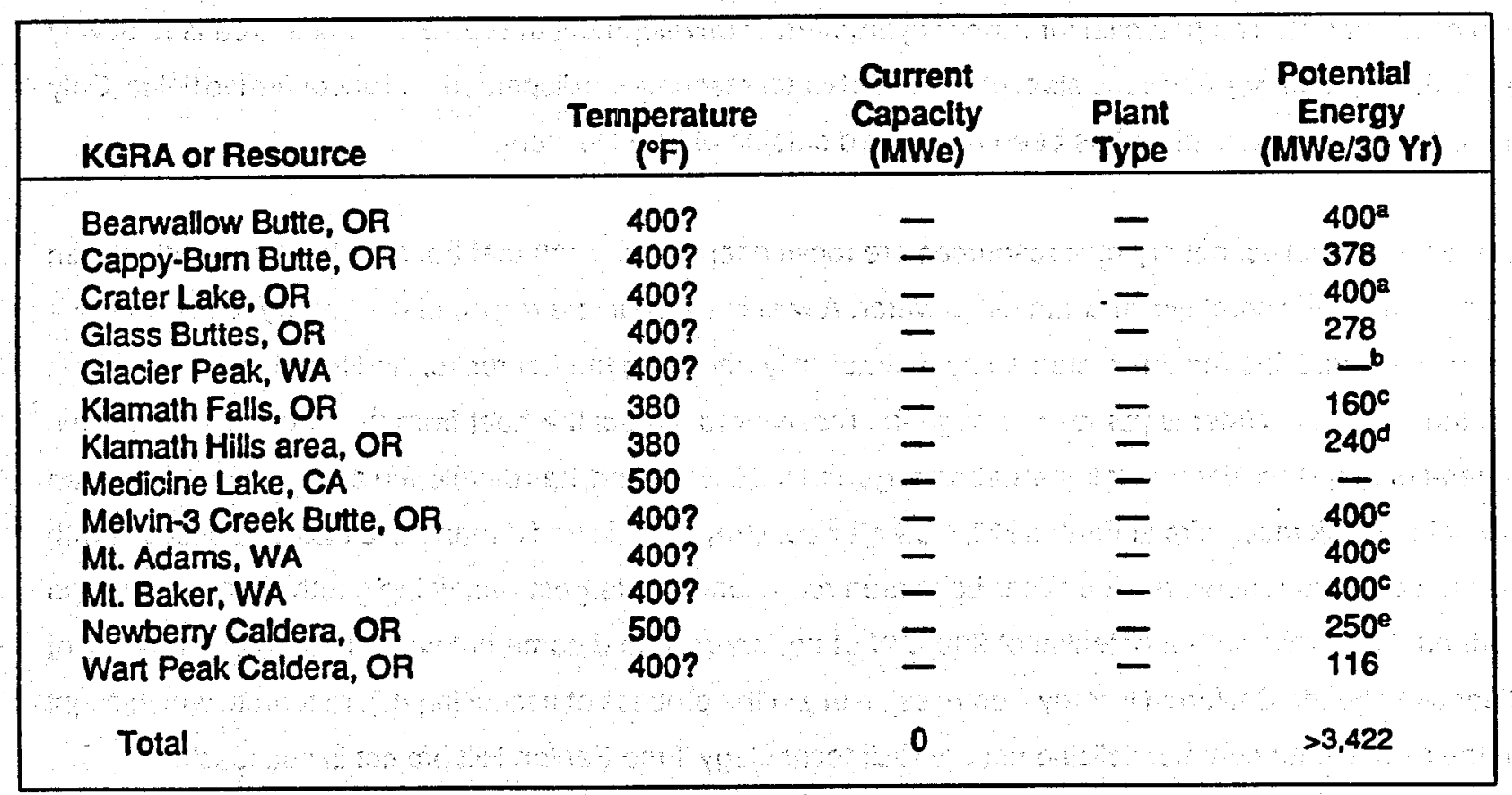

Note: USGS Report 790 did not include electric energy estimates for most of the young volcanics in the Cascades. Unless othenwise noted, temperatures and reservoir potential estimates are from PG\&E. "Prepared testimony of the Informational Hearing," Energy Conservation Resource Development Commission. 1989.

- Largest potential estimate used in PG\&E. "Prepared testimony"; resource may be larger.

bUSGS Rpt. 790 estimates resource energy $=27 \mathrm{MWe} B 0$ yr and temperature $=329^{\circ} \mathrm{F}$.

CUSGS Rpt. 790 estimates resource energy $=.500 \mathrm{MWe} / 30 \mathrm{yr}$ and temperature $=232^{\circ} \mathrm{F}$

JUSGS Rpt. 790 estimates resource energy $=52 \mathrm{MWe} / 30 \mathrm{yr}$ and temperature $=255^{\circ} \mathrm{F}$.

USGS Rpt. 790 estimated resource energy $=740 \mathrm{MWe} / 30 \mathrm{yr}$ and temperature $=446^{\circ} \mathrm{F}$. Resource energy has also been estimated as $1557 \mathrm{MWe} / 30$ yr and temperature $=496^{\circ} \mathrm{F}$. However, these were before Newberry was declared a national monument, which removed the best geothermal prospect area, the caldera, from development. 
Dry steam reservoirs produce dry or nearly dry steam (without liquid water). Dry steam reservoirs are very rare. The United States Geological Survey (USGS) lists only three dry steam resources in the United States: The Geysers, Mt. Lassen, and Yellowstone National Park.

Hot water reservoirs produce hot water or steamwater mixtures. Fluid temperatures range from less than boiling to $675^{\circ} \mathrm{F}$ at Puna, Hawaii. The liquids usually contain various quantities of dissolved salts, so they are also called brines. The potential for further hydrothermal development in PG\&E's service area is relatively small (300-MW range), and consists of primarily hot water resources in dispersed and unconfirmed fields. Only one 2-MW binary cycle plant has been developed outside of The Geysers.

As the name implies, hot dry rock resources are rocks deep in the earth that become hot from contact with the earth's molten core, which contains no water. A well is drilled into a region of the hot dry rocks, where a reservoir (permeable region) is created by hydraulically fracturing the hot rocks. Additional wells are drilled into the reservoir. Water is pumped through the reservoir to extract the heat from the hot rocks for electric generation. The Los Alamos National Laboratory, under DOEfunding, has developed and successfully tested two hot dry rock reservoirs at Fenton Hill, New Mexico. Great Britain and Japan have had less success with their hot dry rock reservoirs. The Clear Lake area represents the largest remaining geothermal resource in Northern California, with a potential of $900 \mathrm{MW}$ of hot dry rock and some hot water resources. The city of Clearlake and the California Energy Commission are in the process of assessing this resource, which might be the next site for commercializing hot dry rock technology if the Fenton Hill project is successful.

Approximately 20,000 MW of hydrothermal resource potential are thought to exist in the Pacific Northwest, in Southern California, and east of the Sierra Nevada. The Pacific Northwest has not had any development to date. BPA is currently planning three separate $10-\mathrm{MW}$ projects to confirm the resource potential of several large fields. Recent exploration at Medicine Lake, California, near the Oregon border indicates a potentially significant resource is near transmission lines running to Northern California. Resource development east of Sierra Nevada will be limited by transmission access to major power markets, particularly Northern California.

Geopressured geothermal resources are deeply buried (generally $>15,000$ feet), high pressure (generally $>10,000 \mathrm{psia}$ ), hot water hydrothermal reservoirs. Brine temperatures range from $300^{\circ} \mathrm{F}$ to $360^{\circ} \mathrm{F}$ in the Gulf Coast region. The brines also contain dissolved natural gas. Thus, heat, natural gas, and hydraulic energy can be utilized for electric generation., Heat is created by pressure on the brines caused by the weight of the earth's crust above. 
Magma resources are "near-surface" molten, or partially molten, rocks from relatively recent volcanic activity that can be reached with current drilling techniques. Under DOE sponsorship. Sandia National Laboratories is performing magma experiments.

\section{ENVIRONMENTAL IMPACTS}

Compared to most electric generation technologies, geothermal power is environmentally benign, with minimal to no air emissions, water impacts, and land-use impacts. However, many of the best geothermal sites are in environmentally sensitive areas and can be difficult or impossible to develop (e.g., Lassan National Park).

Most geothermal fluids contain some noncondensible gases, including hydrogen sulfide $\left(\mathrm{H}_{2} \mathrm{~S}\right)$ and the "greenhouse gases," carbon dioxide and methane. Several processes have been developed to remove $\mathrm{H}_{2} \mathrm{~S}$. before the other noncondensible gases are released to the atmosphere. These have been installed on the direct steam plants at The Geysers and Cove Fort, Utah. Except at one plant, $\mathrm{H}_{2} \mathrm{~S}$ removal has not been required at hot water geothermal plants to meet air quality regulations. Greenhouse gases are released from dry steam plants, but in very small quantities compared to fossil-fueled powerplants. All of the noncondensible gases can be reinjected at most hot watergeothermal reservoirs with either flash steam or binary powerplants.

Surface water impacts are minimal. The steam condensate from direct steam and flash steam plants is generally used to supply cooling tower water. Waste brines, excess condensate, and waste chemicals for $\mathrm{H}_{2} \mathrm{~S}$ emissions control are reinjected into the geothermal reservoir safely below the potable water table. Binary plants can be air cooled so that surface waters are not required for plant cooling.

Land-use impacts are also minimal. Only a few acres must be dedicated to the power plant, well pads, cooling towers, roads, and geothermal fluid gathering and reinjection lines. Agricultural use and livestock grazing are generally compatible with geothermal power generation. With proper siting, scenic/recreational land uses also can be compatible.

The hot water hydrothermal resources in the Salton Trough in Southern Califomia produce high solid content brines. Silica is removed trom the brines before they are piped into the power plant to control in-plant scaling. Silica disposal is a potential land waste disposal problem. However, beneficial uses, such as road base and brick material, have been developed for the silica.

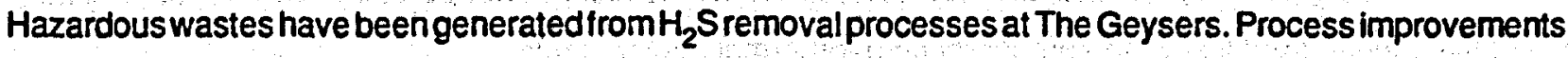
have been developed to minimize hazardous wastes production. Hazardous chemicals may be used for $\mathrm{H}_{2} \mathrm{~S}$ 
emissions control. Drilling wastes, some of which may be hazardous, also require surface disposal. The working fluid used in most binary power plants is flammable.

\section{DEPLOYMENT CONSIDERATIONS AND HURDLES}

Development of a geothermal power plant involves many important issues and considerations. Potential hurdles include public "not in my backyard" reactions, regulatory opposition, lengthy and rigorous permitting processes, environmental issues, transmission access. Independent power producers must also contend with availability of power sales agreements, project financing, and competitive bidding for power.

If third-party financing is required, financial risk can be a major consideration/hurdle. Financial risk includes both development risks and technical risks. Development risks include designehanges, cost overruns, delays, and regulatory changes. The greatest technical risk is that the geothermal resource performance and, consequently, power revenues may be less than predicted.

Underperformance could be due to lower than predicted geothermal fluid temperatures or production rates. A recent steam supply decline at The Geysers Power Plant is an example of underperformance. Too much water reinjection can cause reservoir temperature to drop, affecting power plant performance. Geothermal powergeneration technology is reliable; there is little power plant performance risk. Geothermalfossil hybrids can reduce reservoir risk by guaranteeing a minimum revenue stream from the "fossil side" of the hybrid plant.

\section{TECHNOLOGY TRENDS AND FORECAST}

Geothermal energy conversion technology is relatively mature, but incremental cost and performance improvements can, and will, be made. DOE plans to sponsor an initiative for higher efficiency hydrothermal technologies.

Reservoir management (exploration, development, and operation) requires.significant R\&D, which would address improved techniques to reduce geothermal power project risk, leading to improved project financing availability and lower interest costs. Geothermal reservoir/plant underperformance at The Geysers, Heber, and Larderelio, ltaly, are examples of the need of additional reservoir R\&D. Reservoir risk uncertainty could represent a major hurdle for financing future geothermal projects. In the United States, DOE, the USGS, state governments (including the CEC), and several private firms are sponsoring reservoir research, and it is also underway in many other nations. Reservoir R\&D covers all four types of geothermal resources. However, the geothermal industry can only move up the reservoir exploration/development/operation learning curve by the experience gained from installing and operating geothermal power plants. 
In addition to reservoir risk, cost competition from natural gas-fueled combined cycle power plants will impede the future development of geothermal power. Geothermal power can compete more effectively if its indigenous and low environmental impact attributes are considered in benefit/cost evaluations.

Expansion of geothermal generation from hot water hydrothermal plants will continue on a regional basis through the 1990s. The expansion rate will be impacted by the factors discussed above. Installation and operation of geothermalfossil hybrid power plants can "bridge" over the natural gas supply "bubble" while providing the needed geothermal reservoir management experience. Then, the geothermal industry, including both reservoir management and power generation, will be available and mature when needed by the United States.

There are no known developable new dry U.S. steam resources. Electric generation from hot dry rock may occur early in the next century if the DOE Fenton Hill tests are successful. The CEC is currently sponsoring evaluation of the Clear Lake, hot dry rock resource. Geopressured geothermal resources will probably not be developed for electric generation alone. In the future, electric generation from geopressured geothermal energy may occur in the southwest, including California, in conjunction with thermal enhanced oil recovery. Magma resources are not likely to be exploited commercially for some time.

\section{PG\&E AND UTILITY IMPACTS AND IMPLICATIONS}

Technology improvements are expected to reduce the 1986 life cycle cost of electric energy from hot water hydrothermal resources by $25 \%$ to $35 \%$ by 1997 . Depending on the generation costs and fuel supply of competing technologies, the geothermal cost reduction will increase the supply of economic geothermal capacity in the Western United States. Most of this new generation will be developed to the east (in the Basin and Range) and south (in the Salton Trough) in PG\&E's service area. Because of transmission constraints, etc., it is not clear how PG\&E's ratepayers can benefit from this future low-cost hot water geothermal generation.

The state of California and its utilities have the opportunity to decrease their dependence on conventional fuels by further developing California's geothermal resources. The USGS estimates that over 10,000 MWe of generation are available for 30 years at the major geothermal resources within California in the Salton Trough, and at Coso, Mammoth/Long Valley, Clear Lake, and Medicine Lake.

Power output at The Geysers had dropped from a peak of 1,350 MW (PG\&E-owned units) to around $800 \mathrm{MW}$ by mid-1991 as a result of the steam field decline. Output is forecasted to decline to $400 \mathrm{MW}$ by the mid-1990s and to under $200 \mathrm{MW}$ in the post-2000 time frame. Non-PG\&E-owned Geyser units are experiencing similar 
difficulties. Approaches being investigated to halt the decline include innovative plant operation regimes, new reservoir management techniques, improved reservoir modeling, additional water supply for reinjection, and dry cooling and binary cycle retrofit concepts.

In addition, PG\&E should promote the development of additional low-cost geothermal resources within its service area. The only currently identified significant geothermal resource within PG\&E's service area is at Clear Lake. Present information is that Clear Lake is primarily a hot dry rock resource, although hot water hydrothermal resources may also.exist. The CEC is sponsoring an evaluation of this resource.

\section{PG\&E ADVANCED ENERGY SYSTEMS GEOTHERMAL R\&D PROGRAM}

Geothermal has not currently been targeted as a major opportunity technology for demonstration by PG\&E's R\&D Program because of the relatively mature nature of the hydrothermal technology, the limited potential. for further hydrothermal resource development in its service area and the embryonic state of development of hot dry rock technology.

PG\&E has previously participated in the DOE Heber binary cycle geothermal project and in an evaluation of EPRI's advanced modular wellhead binary system. PG\&E's current technology assessment efforts have focused on characterizing the regional geothemal resources and identifying technology R\&D needs. Modular, higher efficiency binary cycles could help to confirm and more effectively develop the remaining dispersed and small hot water reservoirs in Northern Califomia. HDR development at Clear Lake might represent an opportunity if the Fenton Hill tests are successful. PG\&E R\&D can influence development of the Clear Lake resource as a member of the Phase II Peer Review Group: Hot Dry Rock in the Clear Lake Area. Advanced geothermal and high efficiency natural gas hybrid configurations might offer attractive market features for new hydrothermal resources outside the service area.

Bibliography for Geothermal Energy is on page 279. 


\section{GEOTHERMAL ENERGY}

Altshuler, S. L., and T. D. Arcado. 1989. “Ambient air monitoring at The Geysers: A historical perspective and current status." Geothermal Resources Council Transactions. v. 13. October 1989.

Altshuler, S. L., et al. 1985. "Concentrations of non-criteria air pollutants in the vicinity of The Geysers, California." Geothermal Resources Council Transactions. v. 9, part 1. August 1985.

Amir, E., and M. Ringer. 1987. Relative Cost of Electricity Production. Califomia Energy Commission. April 1987.

Anderson, D. N., ed. 1989. State scene: California and Texas." Geothermal Resources Council Bulletin. v. 18. November 1989.

Annual Report of Pacific Gas and Electric Company to the Public Utilities Commission of the State of Califomia for the year ending December 31, 1988. v. 1. May 1989.

Arcado, T.D., and S. L.Altshuler. 1989. "Ambient air mercury concentrations at The Geysers." Geothermal Resources Council Transactions. v. 13. October 1989.

Austin, A. L. 1975. "Prospects for Advances in Energy Conversion Technologies for Geothermal Energy Development," Proceedings of the SecondU.N. Symposiumonthe Development and Use of Geothermal Resources, San Francisco, May 1975.

Ballantine, D. B. 1990. "The Evolution of Power Plant Design at The Geysers." Geothermal Resources Council Conference.

Bartholomew, D. 1989. Raising $\$ 500$ million on steam in the desert. Corporate Finance. April 1989.

Bemis, G. R. 1989. Technology Characterizations. California Energy Resources Conservation and Development Commission. October 1989.

Bliem, C. J. 1989. Aspects of the Kalina Technology Applied to Geothermal Power Production. EGG-EP-8708. Idaho National Engineering Laboratory. v. 18.

Bloomquist, R. G., et al. 1985. Evaluation and Ranking of Geothermal Resources for Electrical Generation on Electrical Offset in Idaho, Montana, Oregon and Washington. Bonneville Power Administration. Report DOE/BP-13609-1. Portland, OR. June 1985.

Bloomquist, R. G., J. D. Geyer, and B. A. Sifford. 1989. Innovative Design of New Geothermal Generating Plants. DOE/BP-13609-5. July 1989.

Borgo, P. The Potential of Using Geothermal Energy as a Peaking Power Source. Prepared for the U.S. Department of Energy, unpublished.

Brown, R. J., and W. P. Jordan. 1989. "Element content of rainbow trout and Sacramento sucker in streams of The Geysers region, CA." Geothermal Resources Council Transactions. v. 13. October 1989.

Bureau of Land Management. 1986. "Dixie Valley Geothermal Project, Oxbow Geothermal Corp." Environmental Assessment. April 21, 1986. 
-1985. Geothermal Resources Operational Order No. 4. General Environmental Protection Requirements. August 1, 1975.

California Energy Commission. 1990. Docket No.88-ER -8, "Committee Decision on Phase One Resource Assumptions," Appendix A, p. 1, January 5, 1990.

1989. Technology Characterizations. Final Report.

- 1989. Electricity 1988 Report. P106-88-001.

-1988. Energy Technology Status Report.

-1985. Commercial Status of Electrical Generation Technologies.

1984. Technical Assessment Manual.

Power Plant Siting.

Carle, S.F., and N. E. Goldstein. August 1987. "Gravity and fault structures Long Valley Caldera," Geothermal Resources Council Transactions, Vol. 11.

Carrini, D. J., and J. Record. June 1982. "Wellhead power production with a rotary separator turbine." Geothermal Resources Council Conference on Small Scale Geothermal Power Plants.

Cartiere, R. June 22, 1985. Safety rules tightened at The Geysers. Santa Rosa, California: The Press Democrat.

Cedillo, R., and R. N. Yamaoaki. 1981. "The Brawley 10 MWe Power Plant, Unit 1." Geothermal Resources Council Transactions. v. 5.

Code of Federal Regulations. v. 43. October 1986.

DiPippo, R. February 1987. "Geothermal power plants worldwide status - 1986." Proceedings of the Tenth Annual Geothermal Conference and Workshop, Portland, OR. June 24-26, 1986. EPRI AP 5059-SR, 2-6.

1980. Geothermal Energy as a Source of Electricity. U.S. Department of Energy.

- July 1987. "Kakkonda-shizukuishi, a combined geothermal power- and heating-plant," The Geothermal Hotline, vol. 17, no. 1.

Dunn, J. C. 1990. "Overview: Hardrock Penetration." Proceedings of the Geothermal Program Review VIII. San Francisco, California, April 18-20, 1990.

Energy Information Administration. 1989. Inventory of Power Plants in the United States, 1988. Washington, D.C.

Entingh, D. J. 1990. "Geothermal Electric Costed Supply System: Results for Califomia." Meridian Corporation, Alexandria, Virginia.

Environmental Impact Statement, Draft. 1983. Breitenbush II, Geothermal Area., Willamette and Mt. Hood National Forests, Oregon. 
Fesmire, R., and R. Vincent. 1985. "Reliability through design The Santa Fe geothermal plant No. 1." Geothermal Resources Council Transactions. v. 9, part II.

Fletcher, R. D., and A. Chatterjee. 1988. Indigenous Energy Resource Assessment. Pacific Gas and Electric Company. October 7, 1988.

Flynn. T. 1987. Regulatory Approvals for Electric Generating Plants in Nevada. Geothermal Resources Council Short Course No. 1. October 1987.

Fontes, R. A. 1984. "NCPA Geysers geothermal development of integrated steam management system." Geothermal Resources Council Transactions. v. 8. August 1984.

Geothermal Reports. December 1, 1989. November 15, 1989. November 1, 1989. October 16, 1989. August 1, 1989. April 1, 1989. January 16, 1989. September 1, 1988. April 16, 1984.

Geothermal Resources Council Bulletin. January 1985.

Geyer, J. D., L. M. Kellerman and R. G. Bloomquist. Assessment of Geothermal Resources for Electric Generation in the Pacific Northwest. Northwest Power Planning Council, September 1989.

"Geysers promise 3,000 MWe power as world's largest geothermal plant complex." Geothermal Energy. v. 12, no. 2. February 1984.

Gipe, P. 1989. "Ormat Heats Up." Independent Energy. v. 19, no. 8. October 1989.

Glasstone, S. 1982. Energy Deskbook. U.S. Department of Energy.

Goddard, W. B., C. B. Goddard, and D. H. McLain. 1989. "Future Air Quality Maintenance and Improvements Through the Expanded Use of Geothermal Energy." Geothermal Resources Council Transactions. v. 13. October 1989.

Hanley, C. R., et. al. 1986. "Geothermal hazardous materials and waste minimization program." Electric Power Research Institute Ninth Annual Geothermal Conference and Workshop. February 1986.

Hays, L. G., and D. G. Elliott. 1975. "Two-Phase Engine." U.S. Patent No. 3,879,949.

Hayes, Putnam and Bartless, Inc. 1988. "Presenting an objective multi-attribute bidding system." California Utilities Bidding Workshop. December 1988.

Henderson, J. M., and Gary P. Dorighi. 1989. "Operating experiences of converting a company's Geysers unit 15." Liquid Redox Stretford to a LO-CAT(R) $\mathrm{H}_{2} \mathrm{~S}$ Abatement System at Pacific Gas and Electric Conference, Austin, Texas. May 1989.

_. 1989. "Operating Experiences of Converting a Stretford to a LO-CAT(R) $\mathrm{H}_{2} \mathrm{~S}$ Abatement System at Pacific Gas and Electric Company's Geysers Unit 15, " presented at Geothermal Resources Industry Liquid Redox Conference, Austin, TX, May 1989.

Hibara, Y., M. Ikegami, and K. Obinnata. 1988. "Short cut to economical geothermal power generation with wide range portable turbines." Geothermal Resources Council Transactions. v. 12.

Hibara, Y., M. Ikegami, and T. Kondo. "Advanced technology for geothermal turbines." Geothermal Resources Council Transactions. v. 9, part II. 
Hibara, Y., M. Ikegami, and S. Saito. 1986. "The Heber double flash geothermal power plant." Geothermal Resources Council Transactions. v. 10.

Hinds, A. T. 1982. "Geothermal planning: a tale of two counties." Geothermal Resources Council Transactions. v. 6.

Hinrichs, T. C., and B. W. Dambly. 1980. "East Mesa magmamax power process geothermal generating plant a preliminary analysis." Proceedings of the Fourth Annual EPRI Geothermal Conference and Workshop.

Hirtz, P., and T. MacPhu. 1989. "Development of a safer and more efficient method for abatement of $\mathrm{H}_{2} \mathrm{~S}$ during geothermal well drilling." Geothermal Resources Council Transactions. v. 13.

Hochstein, M. P. 1988. "Assessment and modelling of geothermal reservoirs (small utilization schemes)," Geothermics, vol. 17, no. 1, 1988.

Hodgson, S. F., ed. December 1989. "The Geothermal Hotline," Califomia Division of Oil and Gas.

Holt, B., and R. Campbell. 1984. "The mammoth geothermal project." Geothermal Resources Council Transactions. v. 8.

Intemational Energy Agency. 1987. Renewable Sources of Energy. OECD Publications.

Jordan, W. P. and J. R. Brown. 1989. *A review of the Squaw Creek aquatic monitoring program, The Geysers, California." Geothermal Resources Council Transactions. v. 13.

Kaplan, U., Z. Krieger, and A. Dillenberger. 1987. "Innovative design, construction and operation of the Steamboat 5-MW geothermal powerplant at Steamboat Springs, Washoe County, Nevada." Geothermal Resources Council Transactions. v. 11.

Kestin, J. ed. 1980. "Sourcebook on the Production of Electricity from Geothermal Energy." U.S. Department of Energy. March 1980.

Kerna, M. J., and T.S. Allen. 1984. "Roosevelt Hot Springs unit development - a case history." Geothermal Resources Council Transactions. v. 8.

Koenig, J. B. 1989. "Critique of the geothermal exploration R\&D program." Proceedings of the Geothermal Program Review VII. San Francisco, CA. March 21-23, 1989.

Lam, L., et al. 1989. "Results on upstream removal of impurities from hashed geothermal steam." Proceedings: Eleventh EPRI Geothermal Conference and Workshop. EPRI GS-6380.

Lienau, P. J. and B. C. Lunis. eds. 1989. Geothermal Direct Use Engineering and Design Guidebook. Geo-Heat Center. U.S. Department of Energy. March 1989.

Lund, J. W. 1989. "Geothermal heat pumps, trends and comparisons." Geo-Heat Center Quarterly Bulletin. Fall 1989.

McClenahan, L. and D. McClain. 1990. "Interagency Cooperation on Geothermal Permitting." Presented to the Interagency Geothermal Coordinating Council.

Meridian Corporation. 1990. DOE Geothermal Power Plants Data Base. April 1990. 
-1987. Review of Intemational Geothermal Activities andAssessments of U.S. Industry Opportunities. v. II. Los Alamos National Laboratory. April 1987.

1987. The Public Utilities Regulatory Policy ACt (PURPA) and U.S. Geothermal Industry: Current Controversies and Trends in Federal and State Implementation. Prepared for DOE San Francisco Operations Office. November 1987.

Moss, W.E., et al. 1982. "The Salton Sea 10 MWe power plant, unit 1." Geothermal Resources Council Transaction. v. 6

Muffler, L. J. P. 1981. "Geothermal Resource Assessment." Geothermal Systems: Principals and Case Histories. John Wiley and Sons, Lid.

National Historic Mechanical Engineering Landmark. 1985. The Geysers Unit No. 1. American Society of Engineers. October 1985.

Negus-de Wys, J. 1989. "Properties of geopressured brines and wells in the Gulf Coast and opportunities for industrialresearch participation." Proceeding of the Geothermal Program Review VII.U.S. Department of Energy. March 21-23, 1989.

Nichols, K. E. 1986. "Wellhead powerplants and operating experience at Wendell Hot Springs." Geothermal Resources Council Transactions. v. 10. September 1986.

Nielson, D. L., et al. 1990. "Management of Geothermal Resources." Proceedings of the Geothermal Program Review VIII. San Francisco, California, April 18-20, 1990.

Oman, H. 1986. Energy Systems Engineering Handbook. Englewood Cliffs, New Jersey: Prentice-Hall, Inc.

Oregon Department of Energy. Guide to Oregon's Environmental Permits for Biomass Energy Projects. Contract No. C50065. October 1984.

Social Cost of Energy Generation. Report 007.6-90.3 prepared by Meridian Corporation for the Department of Research and Development. San Ramon, California: Pacific Gas and Electric Company.

Pacific Gas and Electric Company. 1989. "Prepared Testimony of the Informational Hearing, September 21, 1989. In the Matter of: Geysers KGRA Generating Capacity and Steam Resources." Docket No. 89-INF-1. Energy Conservation Resource Development Commission, State of California.

—. 1988. Indigenous Energy Resource Assessment.

- 1987. Common Forecasting Methodology 7. v. III. Electricity Supply Forms 1987-1999. September 1987.

Priest, G. R., and G. L. Black. September 1989. "Geothermal Exploration in Oregon, 1988," Oregon Geology, Vol. 51, No. 5,

Ponder, S.H., and P. Blaydes. 1987. "The future of geothermal development-ominous trends." Geothermal Resources Council Transactions, v. 11.

Ram, H., and Y. Yahalom. 1988. "Commercially successful large scale binary applications." Geothermal Resources Council Bulletin. May 1988. 
Ram, H., and Z. Krieger. "Innovative geothermal power plants: The solution to geothermal resource constraints - the Ormat way." Geothermal Resources Council Transactions. v. 13. 1989.

Renewable Energy Institute. 1986. Annual Renewable Energy Technology Review-Progress Through 1984.

Royce, B. A. 1985. An Analysis of Environmental Regulations Governing the Disposal of Geothermal Wastes in California. Brookhaven National Laboratory. September 1985.

Sagar, R. S., and S. E. Schumacher. 1986. "The Vulcan/BN geothermal power plant, Imperial County, California." BN Geothermal and Magma Power Co. paper. August 1986.

Schoonmaker, J. L. 1989. "The Coso geothermal power projects." Geothermal Resources Council Transactions. v. 12.

Shackleford, B.W. 1987. "The Geysers: meeting the environmental and operating challenges of geothermal power." Proceedings: Ninth Annual and Second IIE-EPRI Geothermal Conference and Workshop. v. 2. August 1987.

Snetsinger, David. 1989. "Hazardous Waste Management." Panel on Water, Geothermal Resources Council Hazardous Waste Management Course, Santa Rosa, California. September 30, 1989.

Sorey, M. L. September 1987. "Overview of the Long Valley Hydrothermal System," Proceedings of the Symposium on the Long Valley Caldera: a Pre-Drilling Data Review, Lawrence Berkeley Laboratory, LBL-23940.

1985. "Present state of the Hydrothermal System in Long Valley Caldera, California," Geothermal Resources Council Transactions, Vol. 9, Part I, August 1985.

Suemnicht, G. A., and R. J. Varga. 1987. "Constraints on Models of Structure and Hydrothermal Circulation in Long Valley Caldera, California," Proceedings of the Symposium on the Long Valley Caldera: $A$ Pre-Drilling Data Review. Lawrence Berkeley Laboratory. September 1987.

Summit of the Arch, Economic Declaration. Paris, France. July 16, 1989.

Tester, J. W., D. W. Brown, and R. M. Potter. 1989. Hot Dry Rock Geothermal Energy -A New Energy Agenda for the 21st Century. LA-11514-MS. Los Alamos National Laboratory.

Tetra Tech, Inc. 1979. Energy Fact Book. U.S. Department of the Navy.

The Energy Report. May 11, 1987.

Tucker, R. E., and R. F. Ward. 1982. "Occidental geothermal No. 1, an innovative plant design." Geothermal Resources Council Transactions. v. 6. October 1982.

U.S. Department of Energy. 1990. Geothermal Energy Program Summary Volume 1: Overview Fiscal Year 1989.

1990. "National Energy Strategy and the Role of Geothermal Technology Development:." Proceedings of the Geothermal Program Review VIII. San Francisco, Califomia, April 18-20, 1990. 
- 1989. Characterization of U.S. Energy Resources and Reserves. Prepared by Meridian Corporation for the U.S. Department of Energy. DE-AC01-86CE30844.

- 1989. Geothermal Progress Monitor No. 11. Washington, D.C. December 1989.

- 1989. Programmatic Objectives of the Geothermal Technology Division. v. 1. May 1989.

- 1989. Geothermal Energy Program Summary, Volume 1: Overview Fiscal Year 1988.

—_. 1987. Equipment and Services for Worldwide Applications. DOE/D-101 30. August 1987.

—. 1987. Geothermal Progress Monitor No. 10. Washington, D.C. July 1987.

-1985. Geothermal Progress Monitor No. 9. Washington, D.C. June 1985.

U.S. Environmental Protection Agency. 1987. Report to U.S. Congress, Management of Wastes from the Exploration, Development, and Production of Crude Oil, Natural Gas, and Geothermal Energy. v. 2 of 3: Geothermal Energy. EPA530-SW-88-003. Office of Solid Waste and Emergency Response. December 1987.

U.S. Geological Survey. 1982. Assessment of Low Temperature Geothermal Resources of the United States, Circular 892. U.S. Department of Interior.

_ 1978. Assessment of Geothermal Resources of the United States, Circular 790. U.S. Department of Interior.

Va San, S., and J. A. Anderson. 1987. "Recent developments in abatement technology of geothermal power plants." Geothermal Resources Council Transactions. v. 11.

Wahl, E. F. 1977. Geothermal Energy Utilization. John Wiley \& Sons, Inc.

White, T. 1987. "Strategic planning for geothermal resource confirmation in the Pacific Northwest." Proceedings of the Tenth Annual Geothermal Conference and Workshop, Portland, OR. June 24-26, 1986. EPRI AP-5059-SR, 9-6. February 1987.

Willett, R. F., et al. 1984. "Heber double-flash geothermal power plant, a fast track project." Electric Power Research Institute, Proceedings: 8th Annual Geothermal Conference and Workshop. June 1984.

Williams, R. 1987. "Pacific Gas and Electric Company geothermal operation at The Geysers - 1986 performance perspective." Proceeding of the Tenth Annual Geothermal Conference and Workshop. Portland, OR. June 24-26, 1986. EPRI AP-5059-SR, 5-69. February 1987.

Williams, S., and K. Porter. 1989. Power Plays. Investor Responsibility Research Center.

Yahalom, Y. 1986. "Design and Application Considerations of the Ormesa $30 \mathrm{MWe}$ Geothermal Power Plant at East Mesa, Imperial County, California," Geothermal Resources Council Bulletin (Sept/Oct 1986). 